\title{
FABER AND GRUNSKY OPERATORS CORRESPONDING TO BORDERED RIEMANN SURFACES
}

\author{
MOHAMMAD SHIRAZI
}

\begin{abstract}
Let $\mathfrak{R}$ be a compact Riemann surface of finite genus $\mathfrak{g}>0$ and let $\Sigma$ be the subsurface obtained by removing $n \geq 1$ simply connected regions $\Omega_{1}^{+}, \ldots, \Omega_{n}^{+}$from $\mathfrak{R}$ with non-overlapping closures. Fix a biholomorphism $f_{k}$ from the unit disc onto $\Omega_{k}^{+}$for each $k$ and let $\mathbf{f}=\left(f_{1}, \ldots, f_{n}\right)$. We assign a Faber and a Grunsky operator to $\mathfrak{R}$ and $\mathbf{f}$ when all the boundary curves of $\Sigma$ are quasicircles in $\mathfrak{R}$. We show that the Faber operator is a bounded isomorphism and the norm of the Grunsky operator is strictly less than one for this choice of boundary curves. A characterization of the pull-back of the holomorphic Dirichlet space of $\Sigma$ in terms of the graph of the Grunsky operator is provided.
\end{abstract}

\section{INTRODUCTION AND MAIN RESULTS}

Let $\mathbb{C}$ denote the complex plane, let $\mathbb{D}$ denote the open unit disc in $\mathbb{C}$, and let $\overline{\mathbb{C}}=\mathbb{C} \cup\{\infty\}$ denote the Riemann sphere. Let also $\mathbb{D}^{-}=\{z \in \mathbb{C}:|z|>1\} \cup\{\infty\}$, and let $\mathbb{S}^{1}$ be the unit circle in $\mathbb{C}$. We use $\mathfrak{g}$ for the genus of a Riemann surface to distinguish it from its Green's function $g$.

Let $\Sigma$ be a bordered Riemann surface (in the sense of L. V. Ahlfors and L. Sario [1. II. 3A]) obtained from a compact Riemann surface $\mathfrak{R}$ of genus $\mathfrak{g} \geq 0$ from which $n \geq 1$ simply connected domains $\Omega_{1}^{+}, \ldots, \Omega_{n}^{+}$, with non-overlapping closures removed. We assume the boundary curves $\Gamma_{1}, \ldots, \Gamma_{n}$ (of $\Sigma$ ) are homeomorphic to $\mathbb{S}^{1}$. We call $\Sigma$ a bordered surface of genus $\mathfrak{g} \geq 0$ with $n$ borders. We further assume that there exist biholomorphisms $f_{k}: \mathbb{D} \rightarrow \Omega_{k}^{+}, k=1, \ldots, n$. The map $f_{k}$ may be normalized by assuming $f_{k}(0)=p_{k}$ for some point $p_{k} \in \Omega_{k}^{+}$. Moreover, we assume each $f_{k}$ admits a quasiconformal extension to an open neighbourhood of $\mathbb{D}$. In this case $\Gamma_{k}=f_{k}\left(\mathbb{S}^{1}\right)$ is a quasicircle in $\mathfrak{R}$ (see Section 2.2 for a definition). Define $\Omega_{k}^{-}=\mathfrak{R} \backslash \operatorname{cl}\left(\Omega_{k}^{+}\right)$, where by cl we mean the closure in the topology of $\mathfrak{R}$. It is clear that $\Omega_{k}^{-}$includes $\Sigma$. Let $\mathbf{f}=\left(f_{1}, \ldots, f_{n}\right), \mathcal{O}=\bigcup_{k=1}^{n} \Omega_{k}^{+}$, and $\Gamma=\bigcup_{k=1}^{n} \Gamma_{k}$.

In this paper 1 we will define the Faber and Grunsky operators corresponding to the surface $\mathfrak{R}$ and $\mathbf{f}$ assuming that $\mathfrak{g}>0$. These operators were defined in the case $n>1$, and $\mathfrak{g}=0$, by D. Radnell, E. Schippers, and W. Staubach 8. They used these two operators to define a period map on the Teichmüller space of such a surface. Also in the case $n=1$, and $\mathfrak{g}>0$, D. Radnell, E. Schippers, W. Staubach,

Received by the editors April 6, 2020, and, in revised form, July 21, 2020.

2020 Mathematics Subject Classification. Primary 30F15; Secondary 30C35.

Key words and phrases. Faber operator, Grunsky operator, Schiffer operator, bordered Riemann surfaces, quasicircles.

${ }^{1}$ This paper contains part of the author's Ph.D. thesis results submitted at the University of Manitoba. 
and the author defined the Faber, Grunsky (and Schiffer) operators corresponding to $\Sigma$ in [7].

The main results in this paper are the following two. First, we will show that the Faber operator (as an operator on some Dirichlet spaces) is a bounded isomorphism when all the boundary curves are quasicircles (Corollary 4.5); also for this choice of boundary curves the Grunsky operator (between some Dirichlet spaces) is a bounded operator of norm strictly less than one (Theorem 5.9). Secondly, we will provide a characterization of the pull-back of $\mathcal{D}(\Sigma)$ (the holomorphic Dirichlet space of $\Sigma$ ) under $\mathbf{f}$ as the graph of the Grunsky operator (Theorem 6.1).

To motivate the topic, we recall the classical Grunsky coefficients and Grunsky operator and some of their relationships here. Let

$$
g(z)=z+\sum_{n=0}^{\infty} b_{n} z^{-n}
$$

be an analytic function in some neighbourhood of $\infty$ (in the Riemann sphere) and one-to-one for $|z|>\rho$ for some $\rho>0$. The coefficients $\left\{c_{k l}\right\}$ defined by

$$
\log \frac{g(z)-g(\zeta)}{z-\zeta}=-\sum_{k=1}^{\infty} \sum_{l=1}^{\infty} c_{k l} z^{-k} \zeta^{-l}
$$

are called the Grunsky coefficients of $g$. Furthermore, if $g(\infty)=\infty$ with residue one, the so-called Grunsky inequalities for $g$, are as follows:

$$
\begin{aligned}
& \sum_{k=1}^{\infty} k\left|\sum_{l=1}^{\infty} c_{k l} \lambda_{l}\right|^{2} \leq \sum_{k=1}^{\infty} \frac{\left|\lambda_{k}\right|^{2}}{k}, \\
& \left|\sum_{k=1}^{\infty} \sum_{l=1}^{\infty} c_{k l} \lambda_{k} \lambda_{l}\right| \leq \sum_{k=1}^{\infty} \frac{\left|\lambda_{k}\right|^{2}}{k},
\end{aligned}
$$

for arbitrary complex variables $\lambda_{k}$ 's, provided that the right hand side series converges.

The Grunsky operator, corresponding to the map $g$, is defined by

$$
\begin{aligned}
G r_{g}: \ell^{2}(\mathbb{C}) & \rightarrow \ell^{2}(\mathbb{C}) \\
\left(x_{k}\right) & \rightarrow\left(\sum_{l=1}^{\infty} \sqrt{k l} c_{k l} x_{l}\right) .
\end{aligned}
$$

It was shown that (see Ch. Pommerenke [6]) the Grunsky inequalities have the following operator theoretic forms:

$$
\left\|G r_{g} x\right\| \leq\|x\|, \quad\left|\left\langle x, G r_{g} x\right\rangle\right| \leq\|x\|^{2} .
$$

Therefore, the Grunsky operator is a bounded operator of norm less than or equal to one for $g$ defined above. It has been known that when the domain $g\left(\mathbb{D}^{-}\right)$is a quasidisc the Grunsky operator norm is strictly less than one.

Some applications of the Grunsky inequalities in the Teichmüller spaces date back to the 1980s; I. V. Žuravlev [23] seems to be the first to work in this subject. Another early work is H. Shiga [18] who used the Grunsky inequalities to investigate the boundaries of Teichmüller spaces. More recently, L. A. Takhtajan and L.-P. Teo 22], and then later Y. Shen [20, defined a map on the universal Teichmüller space 
using the Grunsky operator; see also Y. Shen 21] for defining a Grunsky map on the asymptotic universal Teichmüller space using the Grunsky operator. A question that naturally arises here is how the Faber and Grunsky operators defined in this paper can be used to investigate some properties of the Teichmüller space of $\Sigma$. This is a motivation for the results of the paper.

Outline. In Section 2 we go through basic definitions and theorems of function theory on Riemann surfaces. In Section 3 we define the ingredients required to define the Faber and Grunsky operators on the Riemann surface $\mathfrak{R}$ described above. In Section 4 we define the Faber operator and show some of its properties. In Section 5 we define the Grunsky operator and prove that its norm is strictly less than one when all the boundary curves are quasicircles. In Section 6 we provide a characterization of the pull-back of $\mathcal{D}(\Sigma)$ under $\mathbf{f}$.

Remark 1.1. In the case $n=1$ we simply use $\Omega$, and $\Gamma$ instead of $\Omega_{1}^{+}$and $\Gamma_{1}$, respectively. Therefore, $\mathcal{O}=\Omega$ is simply connected, and $\Sigma=\Omega^{-}$. If $\mathfrak{R}$ is a compact Riemann surface which is in general divided by a Jordan curve $\Gamma$ into two connected subsurfaces, then we use $\Sigma_{1}$ and $\Sigma_{2}$ to indicate these subsurfaces; that is, $\Sigma_{i}$ 's are not necessarily simply connected subsurfaces.

\section{PRELiminaries}

By a domain on a Riemann surface $\mathfrak{R}$ we mean an open connected subset of $\mathfrak{R}$. For a complex-valued function $h$ on a domain, by $\bar{h}$ we mean $\bar{h}(z)=\overline{h(z)}$ for every $z$ in that domain. We use $[h]_{A}\left([\alpha]_{A}\right)$ to indicate the restriction of the function $h$ (1-form $\alpha$ ) to a set $A$.

2.1. Harmonic functions, forms, and Dirichlet spaces. Here we review some standard definitions on a Riemann surface $\mathfrak{R}$ with atlas $\{(U, \phi)\}$ of holomorphic charts; see H. L. Royden [9] or H. M. Farkas and I. Kra [4 for more detail.

For a holomorphic chart $(U, \phi)$ the map $z=\phi(w), w \in U$ is called a uniformizer.

Let $\Sigma$ be a domain of $\mathfrak{R}$, which in some cases could be $\mathfrak{R}$. Given a 1 -form $\alpha=a d x+b d y$ on $\Sigma$ the dual of $\alpha$ is defined by

$$
\star \alpha=\star(a d x+b d y)=a d y-b d x,
$$

which is easily seen to be independent of the choice of coordinates. The conjugate of $\alpha$ is defined by $\bar{\alpha}=\bar{a} d x+\bar{b} d y$. A 1 -form $\alpha$ is called harmonic on $\Sigma$ if both $\alpha$ and $\star \alpha$ are closed 1-forms (i.e., $d \alpha=d \star \alpha=0$ ).

The operators $\partial$ and $\bar{\partial}$ denote the Wirtinger derivatives where the output is understood to be a 1 -form. That is, for a function $f(z)=f(x+i y)$ with continuous first order partial derivatives in $\Sigma$, one has

$$
\begin{array}{lll}
\frac{\partial f}{\partial z}:=\frac{1}{2}\left(\frac{\partial f}{\partial x}-i \frac{\partial f}{\partial y}\right), & \partial f:=\frac{\partial f}{\partial z} d z, \\
\frac{\partial f}{\partial \bar{z}}:=\frac{1}{2}\left(\frac{\partial f}{\partial x}+i \frac{\partial f}{\partial y}\right), & \bar{\partial} f:=\frac{\partial f}{\partial \bar{z}} d \bar{z} .
\end{array}
$$

We use $\partial_{z}$ or $\bar{\partial}_{z}$ when derivatives are taken with respect to a specific variable $z$. Let $d$ denote $\partial+\bar{\partial}$.

A function $u$ is said to be harmonic on $\Sigma$ if $u$ is $C^{2}$ in $\Sigma$ and $d \star d u=0$ (the Laplace equation). 
On the vector space of all harmonic 1-forms on $\Sigma$, we define the following inner product and subspaces:

$$
\begin{aligned}
\|\alpha\|_{A_{\text {harm }}(\Sigma)} & =\frac{1}{2} \iint_{\Sigma} \alpha \wedge \star \bar{\alpha}, \quad \alpha \text { harmonic 1-form on } \Sigma, \\
A_{\text {harm }}(\Sigma) & :=\left\{\alpha: \alpha \text { is a harmonic 1-form on } \Sigma \text { and }\|\alpha\|_{A_{\text {harm }}(\Sigma)}<\infty\right\}, \\
\mathcal{D}_{\text {harm }}(\Sigma) & :=\left\{h: h \text { is a harmonic function on } \Sigma, d h=\partial h+\bar{\partial} h \in A_{\text {harm }}(\Sigma)\right\} .
\end{aligned}
$$

We call $A_{\text {harm }}(\Sigma)$ the Bergman space of harmonic 1-forms on $\Sigma$ which is a Hilbert space equipped with the inner product

$$
\langle\alpha, \beta\rangle_{A_{\text {harm }}(\Sigma)}=\frac{1}{2} \iint_{\Sigma} \alpha \wedge \star \bar{\beta}, \quad \alpha, \beta \in A_{\text {harm }}(\Sigma),
$$

and $\mathcal{D}_{\text {harm }}(\Sigma)$ the Dirichlet space of harmonic functions on $\Sigma$, or simply the harmonic Dirichlet space of $\Sigma$ which is also a Hilbert space equipped with $\langle h, g\rangle_{\mathcal{D}_{\text {harm }}(\Sigma)}=\langle d h, d g\rangle_{A_{\text {harm }}(\Sigma)}$.

Let $A(\Sigma)$ denote the subspace of holomorphic 1-forms in $A_{\text {harm }}(\Sigma)$. Similarly, $\overline{A(\Sigma)}$ contains all the anti-holomorphic ones. We naturally have

$$
A_{\text {harm }}(\Sigma)=A(\Sigma) \oplus \overline{A(\Sigma)} \text {. }
$$

We use $\mathcal{D}_{\text {harm }}(\Sigma)_{q}$ to indicate the set of functions in $\mathcal{D}_{\text {harm }}(\Sigma)$ which vanish at $q \in \Sigma . \mathcal{D}(\Sigma)_{q}$ and $\overline{\mathcal{D}(\Sigma)_{q}}$ are defined similarly. $A_{\text {harm }}(\Sigma)_{e}$ contains the exact elements in $A_{\text {harm }}(\Sigma)$; that is, if $\alpha \in A_{\text {harm }}(\Sigma)_{e}$, then there exists $h \in \mathcal{D}_{\text {harm }}(\Sigma)$ such that $d h=\alpha \cdot A(\Sigma)_{e}$ and $\overline{A(\Sigma)}$ are defined in the same way.

If $\alpha$ is an element in $A_{\text {harm }}(\Sigma)$, then the Dirichlet semi-norm of $\alpha$ is defined by

$$
\|\alpha\|_{A_{\text {harm }}(\Sigma)}^{2}:=\langle\alpha, \alpha\rangle_{A_{\text {harm }}(\Sigma)} .
$$

Similarly, for an element $h \in \mathcal{D}_{\text {harm }}(\Sigma)$, we have

$$
\|h\|_{\mathcal{D}_{\text {harm }}(\Sigma)}:=\|d h\|_{A_{\text {harm }}(\Sigma)} .
$$

This implies that the operators

$$
\begin{aligned}
\partial: \mathcal{D}(\Sigma) & \rightarrow A(\Sigma)_{e} \\
h & \rightarrow \partial h
\end{aligned}
$$

and

$$
\begin{aligned}
\bar{\partial}: \overline{\mathcal{D}(\Sigma)} & \rightarrow \overline{A(\Sigma)}_{e} \\
\bar{h} & \rightarrow \bar{\partial} \bar{h}
\end{aligned}
$$

preserve the Dirichlet semi-norm.

Remark 2.1. $\partial$ and $\bar{\partial}$ are isomorphisms if restricted to $\mathcal{D}(\Sigma)_{q}$ and $\overline{\mathcal{D}(\Sigma)_{q}}$ for some $q \in \Sigma$, respectively.

Suppose $A$ and $B$ are two domains in some Riemann surfaces and $\phi: A \rightarrow B$ is a biholomorphism. It is easy to show that $h \in \mathcal{D}_{\text {harm }}(B)$ if and only if $h \circ \phi \in$ $\mathcal{D}_{\text {harm }}(A)$. In particular, the composition with $\phi$ (i.e., pull-back by $\phi$ ) preserves the Dirichlet semi-norm. Thus, the composition operator

$$
\begin{aligned}
\mathcal{C}_{\phi}: \mathcal{D}_{\text {harm }}(B) & \rightarrow \mathcal{D}_{\text {harm }}(A) \\
h & \rightarrow h \circ \phi
\end{aligned}
$$

is an isometric isomorphism (with respect to Dirichlet semi-norm). 
Every harmonic function $h \in \mathcal{D}_{\text {harm }}(G)$ on a simply connected domain $G$ (on a Riemann surface), can be decomposed as $h_{1}+\bar{h}_{2}$ for some $h_{1}, h_{2} \in \mathcal{D}(G)$ where $h_{1}$ and $h_{2}$ are determined uniquely by the condition that $h_{1}(p)=0$ for some $p \in G$. Define the holomorphic projection operator

$$
P_{G}: \mathcal{D}_{\text {harm }}(G) \rightarrow \mathcal{D}(G)_{p}
$$

taking $h$ to $h_{1}$. Similarly, define the anti-holomorphic projection operator

$$
\bar{P}_{G}: \mathcal{D}_{\text {harm }}(G) \rightarrow \overline{\mathcal{D}(G)}
$$

taking $h$ to $\bar{h}_{2}$. In particular, by $P_{\mathbb{D}}$ and $\bar{P}_{\mathbb{D}}\left(P_{\mathbb{D}}-\right.$ and $\left.\bar{P}_{\mathbb{D}^{-}}\right)$we mean the projection operators for $\mathbb{D}\left(\mathbb{D}^{-}\right)$in $\overline{\mathbb{C}}$ where $p=0(p=\infty)$. Note that $P_{G}(a)=0$ and $\bar{P}_{G}(a)=a$ for any constant $a \in \mathbb{C}$. It is clear that we have the identity

$$
I=P_{G}+\bar{P}_{G},
$$

where $I$ is the identity operator on $\mathcal{D}_{\text {harm }}(G)$. We may need to project a harmonic 1-form on a given domain $G$ (not necessarily simply connected) to its holomorphic and anti-holomorphic parts. By $P(G)$ and $\bar{P}(G)$ we mean the projection operators of harmonic 1-forms on $G$ to the holomorphic and the anti-holomorphic parts, respectively.

2.2. Transmission and bounce operators, and CNT boundary limits. Definitions and notation in this section are taken from recent papers of E. Schippers and W. Staubach [16,17]. Let $\mathfrak{R}$ be a compact Riemann surface. Then we have the following definitions:

- A Jordan curve in $\mathfrak{R}$ is a homeomorphic image of $\mathbb{S}^{1}$.

- An open connected subset $G$ of $\mathfrak{R}$ containing a Jordan curve $\Gamma$ is called a doubly connected neighbourhood of $\Gamma$ in $\mathfrak{R}$ if $G$ is bounded by two nonintersecting Jordan curves, each one homotopic to $\Gamma$ within the closure of $G$.

- The pair $(G, \phi)$ where $G$ is as above and $\phi: G \rightarrow \mathbb{A}$ is a biholomorphism to some annulus $\mathbb{A}$ in $\mathbb{C}$ is called a doubly connected chart for $\Gamma$. Although we use the same notation here as the one used for a general uniformizer, it should be clear from the context which one is the case.

- Every Jordan curve $\Gamma$ in $\mathfrak{R}$ which has a doubly connected chart is called a strip-cutting Jordan curve.

- A collar neighbourhood of a Jordan curve $\Gamma$ in $\mathfrak{R}$ is an open connected subset $A$ of $\mathfrak{R}$ bordered by $\Gamma$ and $\Gamma^{\prime}$, where $\Gamma^{\prime}$ is a Jordan curve in $\mathfrak{R}$ which is homotopic to $\Gamma$ from within the closure of $A$ and such that $\Gamma \cap \Gamma^{\prime}$ is empty.

- A collar chart for a Jordan curve $\Gamma$ in $\mathfrak{R}$ is a collar neighbourhood $A$ together with a biholomorphism $\phi: A \rightarrow \mathbb{A}$ for some annulus $\mathbb{A}$ in $\mathbb{C}$.

- A Jordan curve $\Gamma$ on a Riemann surface $\mathfrak{R}$ is called a quasicircle if there exists a biholomorphism $\phi: A \rightarrow B$, where $A$ is a doubly connected neighbourhood of $\Gamma$ in $\mathfrak{R}$ and $B$ is a doubly connected domain in $\mathbb{C}$, such that $\phi(\Gamma)$ is a quasicircle in $\mathbb{C}$. See O. Lehto 5 for a definition of a quasicircle in $\mathbb{C}$.

We define the following conformally non-tangential (abbreviated by CNT) boundary limits for harmonic functions defined on simply connected domains of a compact Riemann surface $\mathfrak{R}$. This is similar to the well-known notion of the non-tangential limit of harmonic functions defined on $\mathbb{D}$ in $\mathbb{C}$.

Definition 2.2 (CNT boundary limit). Let $\mathfrak{R}$ be as above and let also $\Omega$ be a simply connected domain of $\Re$ bounded by a Jordan curve $\Gamma$. Let also $s \in \Gamma$ and 
let $f: \mathbb{D} \rightarrow \Omega$ be a biholomorphism map. We say that $h: \Omega \rightarrow \mathbb{C}$ has CNT limit at $s$ if $\mathcal{C}_{f} h=h \circ f$ has non-tangential limit at $f^{-1}(s)$. It can be shown that this is independent of the choice of $f$.

Now suppose $\Gamma$ is a strip-cutting Jordan curve dividing $\mathfrak{R}$ into two connected subsurfaces $\Sigma_{1}$ and $\Sigma_{2}$. Suppose $\Gamma$ is positively oriented with respect to $\Sigma_{1}$. Using Green's function of $\Sigma_{1}$ with singularity at some $q \in \Sigma_{1}$ (see Section [2.3), it was shown in [16] that there exists $A$, a collar neighbourhood of $\Gamma$ in $\Sigma_{1}$, with a biholomorphism

$$
\phi_{0}: A \rightarrow \mathbb{A}
$$

where $\mathbb{A}$ is an annulus in $\mathbb{C}$, such that $\phi_{0}(\Gamma)=\mathbb{S}^{1} . \phi_{0}$ is called the canonical collar chart with respect to $q$ and $\Sigma_{1}$. Now we have the following definition.

Definition 2.3 (Null set). Let $\Gamma$ be as above. A Borel set $I$ in $\Gamma$ is called a null set (with respect to $\Sigma_{1}$ and $q$ ) if $\phi_{0}(I)$ has logarithmic capacity zero in $\mathbb{S}^{1}$.

It can be shown that this definition is independent of the choice of $q \in \Sigma_{1}$. Moreover, when $\Gamma$ is a quasicircle the definition is also independent of the choice of $\Sigma_{1}$. That is, $I$ is a null set with respect to $\Sigma_{1}$ if and only if it is a null set with respect to $\Sigma_{2}$; see [17, Theorems 3.2, 3.3] for a proof.

Lemma 2.4. Let $\mathfrak{R}, \Gamma, \Sigma_{1}, \Sigma_{2}$, and $\phi_{0}$ be as above. Then a finite union of null sets in $\Gamma$ is a null set in $\Gamma$.

The above was proven inside the proof of [17, Theorem 2.14]. Briefly, it follows from the sub-additivity of the outer capacity under countable unions and Choquet's theorem, which says that for bounded Borel sets in $\mathbb{S}^{1}$ the outer capacity is the capacity.

The following theorem shows the existence and uniqueness of the CNT boundary limits for harmonic functions on simply connected domains of $\mathfrak{R}$.

Theorem 2.5 ([3, Theorem 3.2.1]). Let $\mathfrak{R}, \Gamma, \Omega$, and $f$ be the same as in Definition 2.2. Then for every $h \in \mathcal{D}_{\text {harm }}(\Omega), h$ has $C N T$ limit at $s$ for all $s$ except possibly on a null set in $\Gamma$ with respect to $\Omega$. If $h_{1}, h_{2} \in \mathcal{D}_{\text {harm }}(\Omega)$ have the same CNT boundary limits except possibly on a null set in $\Gamma$, then $h_{1}=h_{2}$ on $\Omega$.

For the existence of the CNT boundary limits of a function $h$ in $\mathcal{D}_{\text {harm }}(\Omega)$, under some conditions, $h$ is required to be defined only on a collar neighbourhood of $\Gamma$ in $\Omega$.

Theorem 2.6 ([17, Theorem 3.17]). Let $\mathfrak{R}, \Gamma, \Sigma_{1}$, and $\Sigma_{2}$ be as above. Let $A$ be a collar neighbourhood of $\Gamma$ in $\Sigma_{1}$. Then for any $h \in \mathcal{D}_{\text {harm }}(A)$, $h$ has $C N T$ boundary limits on $\Gamma$ except possibly on a null set in $\Gamma$. Furthermore, there exists a unique $H \in \mathcal{D}_{\text {harm }}\left(\Sigma_{1}\right)$ whose CNT boundary limits agree with those of $h$ except possibly on a null set in $\Gamma$.

Suppose $h_{1} \in \mathcal{D}_{\text {harm }}\left(\Sigma_{1}\right)$ with CNT boundary limit function $H_{1}$ on $\Gamma$. Then a question that naturally arises here is, Is there any function $h_{2} \in \mathcal{D}_{\text {harm }}\left(\Sigma_{2}\right)$ with CNT boundary limit function $H_{2}$ on $\Gamma$ such that $H_{1}=H_{2}$ on $\Gamma$ except possibly on a null set in $\Gamma$ ? It is worth mentioning that for a Jordan curve, the notion of null set may change from one side to the other.

The notation $\mathfrak{O}\left(\Sigma_{1}, \Sigma_{2}\right) h_{1}$ is used for $h_{2}$ if such $h_{2}$ exists and we say $h_{2}$ is the transmission of $h_{1}$ through the Jordan curve $\Gamma$. $\mathfrak{O}\left(\Sigma_{1}, \Sigma_{2}\right)$ is clearly linear on elements in $\mathcal{D}_{\text {harm }}\left(\Sigma_{1}\right)$ for which the transmission exists. 
Example 2.7. If $\mathfrak{R}=\overline{\mathbb{C}}$ and $\Gamma=\mathbb{S}^{1}$, then such a transmission can be written explicitly. That is, for every $h_{1} \in \mathcal{D}_{\text {harm }}\left(\mathbb{D}^{-}\right)$, and $z \in \mathbb{D}$, we have

$$
\left[\mathfrak{O}\left(\mathbb{D}^{-}, \mathbb{D}\right) h_{1}\right](z)=h_{1}\left(\frac{1}{\bar{z}}\right) .
$$

In particular, $\mathfrak{O}\left(\mathbb{D}^{-}, \mathbb{D}\right)\left(1 / z^{n}\right)=\bar{z}^{n}$, where by $1 / z^{n}$ we mean the harmonic function $h_{1}(z)=1 / z^{n}, n \geq 0$. The operator $\mathfrak{O}\left(\mathbb{D}, \mathbb{D}^{-}\right)$on $\mathcal{D}_{\text {harm }}(\mathbb{D})$ can be written similarly.

Remark 2.8. For a polynomial $h \in \mathcal{D}_{\text {harm }}(\mathbb{D})$, the above example and the density of polynomials $\mathbb{C}[z, \bar{z}]$ in $\mathcal{D}_{\text {harm }}(\mathbb{D})$ show that $\mathfrak{O}\left(\mathbb{D}, \mathbb{D}^{-}\right) h$ admits a holomorphic extension to every annulus $\mathbb{A}$ with outer boundary equal to $\mathbb{S}^{1}$.

The work [17, Theorem 3.29] showed the existence and the boundedness of the transmission operator for quasicircles on compact Riemann surfaces. Here is the theorem.

Theorem 2.9. Let $\mathfrak{R}, \Gamma, \Sigma_{1}$, and $\Sigma_{2}$ be as above, where $\Gamma$ is a quasicircle. $\mathfrak{O}\left(\Sigma_{1}, \Sigma_{2}\right)$ is a bounded linear operator from $\mathcal{D}_{\text {harm }}\left(\Sigma_{1}\right)$ onto $\mathcal{D}_{\text {harm }}\left(\Sigma_{2}\right)$ with respect to the Dirichlet semi-norm.

In other words, they proved that for every $h_{1} \in \mathcal{D}_{\text {harm }}\left(\Sigma_{1}\right)$ there exists a unique $h_{2} \in \mathcal{D}_{\text {harm }}\left(\Sigma_{2}\right)$ such that $h_{1}$ and $h_{2}$ have same CNT boundary limits on $\Gamma$ except possibly on a null set in $\Gamma$. A similar result for $\mathfrak{O}\left(\Sigma_{2}, \Sigma_{1}\right)$ is valid due to the symmetry of the definition.

To define the bounce operator let $\mathfrak{R}, \Gamma, \Sigma_{1}$, and $\Sigma_{2}$ be as above. For a given harmonic function $h$ on a collar neighbourhood $A$ of $\Gamma$ in $\Sigma_{1}$, Theorem 2.6 ensures that the following operator is well-defined:

$$
\begin{aligned}
\mathfrak{G}\left(A, \Sigma_{1}\right): \mathcal{D}_{\text {harm }}(A) & \rightarrow \mathcal{D}_{\text {harm }}\left(\Sigma_{1}\right) \\
h & \rightarrow \tilde{h}
\end{aligned}
$$

where $\tilde{h}$ and $h$ have the same CNT boundary limits on $\Gamma$ except possibly on a null set in $\Gamma$.

It was shown that [17, Theorem 3.22] if $\Gamma$ is a quasicircle, then $\mathfrak{G}(A, \Sigma)$ is a bounded operator.

2.3. Cauchy kernel and Green's function on Riemann surfaces. The generalization of the Cauchy kernel to compact (and non-compact) Riemann surfaces is classical; here we follow H. L. Royden [10]. A function $f$ on $\mathfrak{R}$ is said to have a logarithmic pole of mass $m$ at the point $q \in \mathfrak{R}$ if in some holomorphic chart $(U, \phi)$ about $q$ one has

$$
f(p)=-m \log |\phi(p)-\phi(q)|+\psi(p),
$$

where $p \in U$, and $\psi$ is a smooth function on $U$.

Definition 2.10 (Green's function). A function $g$ on $\mathfrak{R}$ which is harmonic on $\mathfrak{R}$ except at the points $q$ and $q_{0}$ and has logarithmic poles there with masses +1 and -1 , respectively, is called Green's function for $\mathfrak{R}$ (with poles at $q$ and $q_{0}$ ).

If such a function exists, then it is unique up to additive constants. Royden, therefore, suggested the notation $g\left(p, p_{0} ; q, q_{0}\right)$ which indicates the normalization in which $g$ is zero at $p_{0} \neq q, q_{0}$. Green's function exists [10, Proposition 1, 2]. We changed the notation to $g(w ; z, q)$ indicating that the poles are at the points $z$ and $q$. 
Consequently, $\partial_{w} g(w ; z, q)$ which has a pole of order one at $z$ and $q$ with residues +1 and -1 , respectively, is our Cauchy kernel. Furthermore, $\partial_{w} g(w ; q, q)=0$.

For a domain $G$ in $\mathfrak{R}$ Green's function can be defined as in the planar case. That is, $g_{G}(., z)$ is called Green's function of $G$ if

- $g_{G}(w, z)$ is a harmonic function in $w$ everywhere on $G \backslash\{z\}$.

- If $(U, \phi)$ is a holomorphic chart containing $z$ in $G$, then $g_{G}(w, z)+\log \mid \phi(w)-$ $\phi(z) \mid$ is harmonic in $w$ for $w \in U$.

- $\lim _{w \rightarrow w_{0}} g_{G}(w, z)=0$ for every $w_{0}$ on the boundary $G$.

Such a function exists; see, e.g., Ahlfors and Sario [1] for a proof of its existence in the case that $\mathfrak{R}$ is compact and no boundary component reduces to a point in $\Re$. Each domain $\Omega_{k}^{+}$has Green's function with singularity at $p_{k} \in \Omega_{k}^{+}$which is denoted by $g_{\Omega_{k}^{+}}\left(., p_{k}\right)$. By $\Gamma_{\epsilon}^{p_{k}}$, we mean the level curves of Green's function of $\Omega_{k}^{+}$; that is,

$$
\Gamma_{\epsilon}^{p_{k}}=\left\{w \in \Omega_{k}^{+}: g_{\Omega_{k}^{+}}\left(w, p_{k}\right)=\epsilon\right\} .
$$

For fixed $k$, these are simple closed analytic curves which approach $\Gamma_{k}$ from within $\Omega_{k}^{+}$as $\epsilon>0$ approaches zero.

2.4. Cauchy-type integral operators. We usually deal with a domain $G$ such that its boundary curve $\Gamma$ is a quasicircle. Since quasicircles might not be rectifiable we cannot calculate a line integral by traversing through $\Gamma$. We, therefore, use limiting integrals in order to define an integral operator on $\Gamma$.

Definition 2.11 (Cauchy-type integral operator). Let $\mathfrak{R}$ be a compact Riemann surface and let $\Gamma$ be a Jordan curve dividing $\mathfrak{R}$ into two connected subsurfaces $\Sigma_{1}$ and $\Sigma_{2}$. Let $p \in \Sigma_{1}$ and $\Gamma_{\epsilon}^{p}$ be the level curves of $g_{\Sigma_{1}}(\cdot, p)$. For a fixed $q \in \mathfrak{R} \backslash \Gamma$, the operator

$$
\begin{aligned}
J_{q}(\Gamma): \mathcal{D}_{\text {harm }}\left(\Sigma_{1}\right) & \rightarrow \mathcal{D}_{\text {harm }}\left(\Sigma_{1} \cup \Sigma_{2}\right)_{q} \\
h & \rightarrow-\lim _{\epsilon \rightarrow 0^{+}} \frac{1}{\pi i} \int_{\Gamma_{\epsilon}^{p}} \partial_{w} g(w ; z, q) h(w),
\end{aligned}
$$

where $z \in \mathfrak{R} \backslash \Gamma$, is called the Cauchy-type integral operator (corresponding to $\Gamma$ ).

Remark 2.12. For a proof of the existence of the above limiting integral, its independence of the choice of $p \in \Sigma_{1}$, and its boundedness see [16, Section 4.1].

The Cauchy-type integral operator for other components of $\mathfrak{R} \backslash \Gamma$ is defined similarly. We use the notation $\left[J_{q}(\Gamma) h\right]_{A}$ or simply $J_{q}(\Gamma)_{A} h$ (or both once we have a double restriction) to indicate that $J_{q}(\Gamma) h$ is restricted to a set $A$.

Remark 2.13. There are some connections between the above Cauchy-type integral operators and the Riemann boundary value problem for quasicircles on Riemann surfaces; see [7, Section 3.2] and the references therein.

We may restrict the Cauchy-type integral operator to holomorphic functions in a collar neighbourhood of a curve $\Gamma$ in $\mathfrak{R}$ [16, Section 4.3 ]. Let $\mathfrak{R}, \Gamma, \Sigma_{1}, \Sigma_{2}$, and $\Gamma_{\epsilon}^{p}$ be as in Definition 2.11 Let $A$ be a collar neighbourhood of $\Gamma$ in $\Sigma_{1}$. Define

$$
\begin{aligned}
J_{q}^{\prime}(\Gamma): \mathcal{D}(A) & \rightarrow \mathcal{D}_{h a r m}\left(\Sigma_{1} \cup \Sigma_{2}\right)_{q} \\
h & \rightarrow-\lim _{\epsilon \rightarrow 0^{+}} \frac{1}{\pi i} \int_{\Gamma_{\epsilon}^{p}} \partial_{w} g(w ; z, q) h(w),
\end{aligned}
$$


where $h \in \mathcal{D}(A)$ and $q \in \mathfrak{R} \backslash \Gamma . \quad J_{q}^{\prime}(\Gamma)$ is defined for holomorphic functions in $A$ whereas $J_{q}(\Gamma)$ is defined for harmonic functions in $\Sigma_{1}$. By the holomorphicity of the integrand the integral is independent of the choice of the analytic curve $\Gamma_{\epsilon}^{p}$ provided that $\epsilon$ is small enough so that $\Gamma_{\epsilon}^{p} \in A$. The following shows a relationship between these two Cauchy-type integral operators.

Theorem 2.14 ([16, Theorem 4.9]). Let $\mathfrak{R}, \Gamma, \Sigma_{1}, \Sigma_{2}$, and $\Gamma_{\epsilon}^{p}$ be as in Definition 2.11. Let $A$ be a collar neighbourhood of $\Gamma$ in $\Sigma_{1}$. If $\Gamma$ is a Jordan curve, then

$$
\left[J_{q}^{\prime}(\Gamma) h\right](z)=\left[J_{q}(\Gamma) \mathfrak{G}\left(A, \Sigma_{1}\right) h\right](z)
$$

for every $z, q \in \mathfrak{R} \backslash \Gamma$.

2.5. Kernel functions on Riemann surfaces. Consider a bounded multiply connected domain $G$ in $\mathbb{C}$ bounded by $n$ pairwise disjoint closed analytic curves with Green's function $g$. M. Schiffer [11] and S. Bergman and M. Schiffer [2] defined the following two kernels, which we call kernel functions:

$$
\begin{aligned}
K(z, \bar{w}) & =-\frac{2}{\pi} \frac{\partial^{2} g(z, w)}{\partial z \partial \bar{w}}, \\
L(z, w) & =-\frac{2}{\pi} \frac{\partial^{2} g(z, w)}{\partial z \partial w} .
\end{aligned}
$$

Analogous to the above, kernel functions may be defined on compact Riemann surfaces. For a compact Riemann surface $\mathfrak{R}(\mathfrak{g}>0)$ with Green's function $g(w ; z, q)$ having singularities at $z$ and $q$, the following two bi-differential forms, also called kernel functions, are well known. The Schiffer kernel is defined by

$$
L_{\mathfrak{R}}(z, w)=\frac{1}{\pi i} \partial_{z} \partial_{w} g(w ; z, q),
$$

and the Bergman kernel is defined by

$$
K_{\mathfrak{R}}(z, w)=-\frac{1}{\pi i} \partial_{z} \bar{\partial}_{w} g(w ; z, q) .
$$

Here are some important properties of these kernels; see M. Schiffer and D. Spencer [13. Chapter 4] or [16, Proposition 3.3] for a proof.

- $L_{\mathfrak{R}}$ and $K_{\mathfrak{R}}$ are independent of the choice of the points $q$ and $w_{0}$.

- $L_{\mathfrak{R}}(z, w)$ is holomorphic in both variables, except for a pole of order two when $w=z$. $K_{\mathfrak{R}}(z, w)$ is holomorphic in $z$ for fixed $w$, and is antiholomorphic in $w$ for fixed $z$.

- $L_{\mathfrak{R}}(w, z)=L_{\mathfrak{R}}(z, w)$, and $K_{\mathfrak{R}}(w, z)=-\overline{K_{\mathfrak{R}}(z, w)}$.

2.6. Schiffer operator. Here we recall the definition of the Schiffer operator and some of its essential properties; see [7] for the Schiffer operators corresponding to the one boundary curve case and [14] for the one corresponding to the many boundary curve case. The work of Schippers and Staubach [16] includes many important facts about this operator including identities involving its adjoint.

We define the Schiffer operator for the many boundary curve case based on the Schiffer operators $T(\Omega ; \Sigma)$ and $T(\Sigma ; \Omega)$ corresponding to the one boundary curve case. 
Let $\mathfrak{R}, \Sigma, \Omega_{k}^{ \pm}$and $\Gamma_{k}, k=1, \ldots, n$, and let $\mathcal{O}$ be the same as in Section 1 . For fixed $j=1, \ldots, n$, the Schiffer operators are defined by

$$
\begin{aligned}
T\left(\Omega_{j}^{+} ; \Omega_{j}^{-}\right): \overline{A\left(\Omega_{j}^{+}\right)} & \rightarrow A\left(\Omega_{j}^{-}\right) \\
\bar{\alpha} & \rightarrow \frac{1}{\pi i} \iint_{\Omega_{j}^{+}, w} \partial_{z} \partial_{w} g(w ; z, q) \wedge \bar{\alpha}(w),
\end{aligned}
$$

where $z \in \Omega_{j}^{-}$, and

$$
\begin{aligned}
T\left(\Omega_{j}^{+} ; \Omega_{j}^{+}\right): \overline{A\left(\Omega_{j}^{+}\right)} & \rightarrow A\left(\Omega_{j}^{+}\right) \\
\bar{\alpha} & \rightarrow \frac{1}{\pi i} \iint_{\Omega_{j}^{+}, w} \partial_{z} \partial_{w} g(w ; z, q) \wedge \bar{\alpha}(w),
\end{aligned}
$$

where $z \in \Omega_{j}^{+}$. The following is, therefore, well-defined.

Definition 2.15 (Schiffer operator for $n>1$ ). Define the Schiffer operator $T(\mathcal{O} ; \Sigma)$ by

$$
T(\mathcal{O} ; \Sigma): \bigoplus_{k=1}^{n} \overline{A\left(\Omega_{k}^{+}\right)} \rightarrow A(\Sigma),
$$

where for $\left(\bar{\alpha}_{1}, \ldots, \bar{\alpha}_{n}\right) \in \bigoplus_{k=1}^{n} \overline{A\left(\Omega_{k}^{+}\right)}$and $z \in \Sigma$ one has

$$
\begin{aligned}
{\left[T(\mathcal{O} ; \Sigma)\left(\bar{\alpha}_{1}, \ldots, \bar{\alpha}_{n}\right)\right](z) } & :=\frac{1}{\pi i} \sum_{k=1}^{n} \iint_{\Omega_{k}^{+}, w} \partial_{z} \partial_{w} g(w ; z, q) \wedge \bar{\alpha}_{k}(w) \\
& =\sum_{k=1}^{n}\left[T\left(\Omega_{k}^{+} ; \Omega_{k}^{-}\right) \bar{\alpha}_{k}\right]_{\Sigma}(z) .
\end{aligned}
$$

For fixed $j=1, \ldots, n$, the Schiffer operator $T\left(\mathcal{O} ; \Omega_{j}^{+}\right)$is defined by

$$
T\left(\mathcal{O} ; \Omega_{j}^{+}\right): \bigoplus_{k=1}^{n} \overline{A\left(\Omega_{k}^{+}\right)} \rightarrow A\left(\Omega_{j}^{+}\right)
$$

where for $z \in \Omega_{j}^{+}$one has

$$
\begin{aligned}
{\left[T\left(\mathcal{O} ; \Omega_{j}^{+}\right)\left(\bar{\alpha}_{1}, \ldots, \bar{\alpha}_{n}\right)\right](z):=} & \sum_{\substack{k=1 \\
k \neq j}}^{n}\left[T\left(\Omega_{k}^{+} ; \Omega_{k}^{-}\right) \bar{\alpha}_{k}\right]_{\Omega_{j}^{+}}(z) \\
& +\left[T\left(\Omega_{j}^{+} ; \Omega_{j}^{+}\right) \bar{\alpha}_{j}\right](z) .
\end{aligned}
$$

Generalizing the notation of [16, Definition 4.17] let us define

$$
\mathcal{V}=\left\{\left(\bar{\alpha}_{1}, \ldots, \bar{\alpha}_{n}\right) \in \bigoplus_{k=1}^{n} \overline{A\left(\Omega_{k}^{+}\right)}: \sum_{k=1}^{n} \iint_{\Omega_{k}^{+}} \beta \wedge \bar{\alpha}_{k}=0 \forall \beta \in A(\Re)\right\} .
$$

The integral condition for elements in $\mathcal{V}$ is equivalent to

$$
<\sum_{k=1}^{n} \bar{\alpha}_{k}, \bar{\beta}>_{A_{h a r m}(\Sigma)}=0
$$

that is, $\sum_{k=1}^{n} \bar{\alpha}_{k}$ is perpendicular to the restriction of elements in $\overline{A(\mathfrak{R})}$ into $\Sigma$. This condition is required for the existence of a solution to the jump problem. See [14, Corollary 2.17] and/or [14, Theorem 2.15] for a proof of the following theorem. 
Theorem 2.16. Let $\mathfrak{R}, \Sigma, \Omega_{k}^{ \pm}$and $\Gamma_{k}, k=1, \ldots, n$, and let $\mathcal{O}$ be the same as in Section 1. The restriction of the Schiffer operator $T(\mathcal{O} ; \Sigma)$ to $\mathcal{V}$ is a bounded isomorphism from $\mathcal{V}$ onto $A(\Sigma)_{e}$.

\section{Some operators AND SUbSPACES OF FUnCTIONS AND 1-FORMS}

We generalize the operators and theorems from one boundary curve case to finitely many ones. Let $\mathfrak{R}, \Sigma, \Omega_{k}^{ \pm}$and $\Gamma_{k}, f_{k}, k=1, \ldots, n$, and let $\mathcal{O}$ be the same as in Section 1. The composition operator for the set of biholomorphisms $\mathbf{f}=\left(f_{1}, \ldots, f_{n}\right)$ is defined by

$$
\begin{aligned}
\widetilde{\mathcal{C}}_{\mathbf{f}}: \bigoplus_{k=1}^{n} \mathcal{D}_{\text {harm }}\left(\Omega_{k}^{+}\right) & \longrightarrow \mathcal{D}_{\text {harm }}(\mathbb{D})^{n} \\
& \left(g_{1}, \ldots, g_{n}\right) \longrightarrow\left(\mathcal{C}_{f_{1}} g_{1}, \ldots, \mathcal{C}_{f_{n}} g_{n}\right),
\end{aligned}
$$

where $\mathcal{C}_{f_{k}}$ is the one defined in Section 2.1

Similar definitions for the inverse functions, $\widetilde{\mathcal{C}}_{\mathbf{f}^{-1}}$, can be given. These operators are clearly bounded isometries with respect to Dirichlet semi-norm.

Definition 3.1. Let $G_{k}, k=1, \ldots, n$, be a collection of pairwise disjoint simply connected domains of a Riemann surface $\mathfrak{R}$. Let $G=\bigcup_{k=1}^{n} G_{k}$. Pick a point $p_{k} \in G_{k}$ for each $k=1, \ldots, n$. Define

$$
\begin{aligned}
\widetilde{P}_{G}: \bigoplus_{k=1}^{n} \mathcal{D}_{h a r m}\left(G_{k}\right) & \longrightarrow \bigoplus_{k=1}^{n} \mathcal{D}\left(G_{k}\right) \\
\left(h_{1}, \ldots, h_{n}\right) & \longrightarrow\left(P_{G_{1}} h_{1}, \ldots, P_{G_{n}} h_{n}\right),
\end{aligned}
$$

where $P_{G_{k}}$ was defined in Section 2.1. In particular, $\left(P_{G_{k}} h_{k}\right)\left(p_{k}\right)=0$.

The projection to the anti-holomorphic part $\widetilde{\bar{P}}_{G}$ is defined similarly. The projections $\widetilde{P}(G)$ and $\widetilde{\bar{P}}(G)$ on harmonic 1-forms may be defined.

Let $A_{k}$ be a collar neighbourhood of $\Gamma_{k}$ in $\Sigma$ and let

$$
\operatorname{Res}\left(\Sigma, A_{k}\right): \mathcal{D}_{\text {harm }}(\Sigma) \rightarrow \mathcal{D}_{\text {harm }}\left(A_{k}\right)
$$

be the restriction operator from $\Sigma$ to $A_{k}$.

Definition 3.2 (Transmission operator). Let $A_{k}$ and Res be as above; then define $\mathfrak{O}\left(\Sigma, \Omega_{k}^{+}\right)$by

$$
\mathfrak{O}\left(\Omega_{k}^{-}, \Omega_{k}^{+}\right) \mathfrak{G}\left(A_{k}, \Omega_{k}^{-}\right) \operatorname{Res}\left(\Sigma, A_{k}\right) .
$$

The transmission operator for $\Sigma$ is defined by

$$
\begin{aligned}
\widetilde{\mathfrak{O}}(\Sigma, \mathcal{O}): \mathcal{D}_{\text {harm }}(\Sigma) & \longrightarrow \bigoplus_{k=1}^{n} \mathcal{D}_{\text {harm }}\left(\Omega_{k}^{+}\right) \\
h & \longrightarrow\left(\mathfrak{O}\left(\Sigma, \Omega_{1}^{+}\right) h, \ldots, \mathfrak{O}\left(\Sigma, \Omega_{n}^{+}\right) h\right) .
\end{aligned}
$$

We generalize the subspace $W$ defined for one boundary curve case in [7, Section 3.2 ] to a many boundary curve case as follows.

Definition 3.3. Define

$$
\mathcal{W}:=\left\{\left(h_{1}, \ldots, h_{n}\right) \in \bigoplus_{k=1}^{n} \mathcal{D}_{h a r m}\left(\Omega_{k}^{+}\right): \sum_{k=1}^{n} \iint_{\Omega_{k}^{+}} \beta \wedge \bar{\partial} h_{k}=0 \forall \beta \in A(\Re)\right\} .
$$


Similarly, the above integral condition can be replaced with

$$
\left\langle\sum_{k=1}^{n} \bar{\partial} h_{k}, \bar{\beta}\right\rangle_{A_{h a r m}(\Sigma)}=0 .
$$

Here is how we define a Cauchy-type integral operator corresponding to $\Omega_{1}^{+}, \ldots, \Omega_{n}^{+}$.

Definition 3.4 (Cauchy-type integral operator). Let $\Re, \Sigma, \Omega_{k}^{ \pm}, \Gamma_{k}, k=1, \ldots, n$, and let $\mathcal{O}$ be the same as in Section 1. Let also $z, q \in \mathfrak{R} \backslash \Gamma$. The Cauchy-type integral operator for domains $\Omega_{1}^{+}, \ldots, \Omega_{n}^{+}$, is defined by

$$
\begin{aligned}
J_{q}(\Gamma): \bigoplus_{k=1}^{n} \mathcal{D}_{\text {harm }}\left(\Omega_{k}^{+}\right) & \rightarrow \mathcal{D}_{\text {harm }}(\mathfrak{R} \backslash \Gamma)_{q} \\
\left(h_{1}, \ldots, h_{n}\right) & \rightarrow \sum_{k=1}^{n}\left[J_{q}\left(\Gamma_{k}\right)_{\Omega_{k}^{-}} h_{k}\right]_{\mathfrak{R} \backslash \Gamma},
\end{aligned}
$$

where

$$
\left[J_{q}\left(\Gamma_{k}\right)_{\Omega_{k}^{-}} h_{k}\right](z)=-\lim _{\epsilon \rightarrow 0^{+}} \frac{1}{\pi i} \int_{\Gamma_{\epsilon}^{p_{k}}} \partial_{w} g(w ; z, q) h_{k}(w) .
$$

Recall $\Gamma$ here is understood as $\bigcup_{k=1}^{n} \Gamma_{k}$. By $\mathcal{D}_{\text {harm }}(\mathfrak{R} \backslash \Gamma)_{q}$ we mean the output of the $J_{q}(\Gamma)$ operator is a harmonic function on $\Sigma$ if $z \in \Sigma$ and is a harmonic function on $\Omega_{k}^{+}$if $z \in \Omega_{k}^{+}$for some $k=1, \ldots, n$. The output does not extend to a continuous function on $\mathfrak{R}$.

Theorem 3.5. Let $J_{q}(\Gamma)$ be defined as above. Then we have

(1) The operator $\left[J_{q}(\Gamma)\right]_{\Sigma}$ is a bounded operator from $\bigoplus_{k=1}^{n} \mathcal{D}_{\text {harm }}\left(\Omega_{k}^{+}\right)$equipped with norm $\|\cdot\|_{\bigoplus_{k=1}^{n} \mathcal{D}_{\text {harm }}\left(\Omega_{k}^{+}\right)}$to $\mathcal{D}_{\text {harm }}(\Sigma)$ equipped with norm $\|\cdot\|_{\mathcal{D}_{\text {harm }}(\Sigma)}$. The same result is true for $\left[J_{q}(\Gamma)\right]_{\Omega_{k}^{+}}, k=1, \ldots, n$.

(2) If the domain of $\left[J_{q}(\Gamma)\right]_{\Sigma}$ is restricted to $\mathcal{W}$, then the output will be a holomorphic function on $\Sigma$. The same result is true for $\left[J_{q}(\Gamma)\right]_{\Omega_{k}^{+}}, k=$ $1, \ldots, n$.

See [16, Corollary 4.3] for a proof of the above. Fix $p_{k} \in \Omega_{k}^{+}$for each $k$ and define

$$
\mathcal{W}^{\prime}=\mathcal{W} \cap \bigoplus_{k=1}^{n} \overline{\mathcal{D}\left(\Omega_{k}^{+}\right)_{p_{k}}}
$$

For notational simplicity we define

$$
K: \mathcal{W}^{\prime} \rightarrow \mathcal{D}(\Sigma)_{q}
$$

by

$$
K\left(\bar{h}_{1}, \ldots, \bar{h}_{n}\right)=\left[J_{q}(\Gamma)\left(\bar{h}_{1}, \ldots, \bar{h}_{n}\right)\right]_{\Sigma}=\sum_{k=1}^{n}\left[J_{q}\left(\Gamma_{k}\right)_{\Omega_{k}^{-}} \bar{h}_{k}\right]_{\Sigma} .
$$

The operator $\widetilde{\partial}$ (similarly $\widetilde{\bar{\partial}}$ ) is defined naturally by

$$
\begin{aligned}
\widetilde{\partial}: \bigoplus_{k=1}^{n} \mathcal{D}_{\text {harm }}\left(\Omega_{k}^{+}\right) & \rightarrow \bigoplus_{k=1}^{n} \mathcal{D}\left(\Omega_{k}^{+}\right) \\
\left(h_{1}, \ldots, h_{n}\right) & \rightarrow\left(\partial h_{1}, \ldots, \partial h_{n}\right) .
\end{aligned}
$$

The proof of the following lemma is straightforward. 
Lemma 3.6. The restriction of the operator $\widetilde{\bar{\partial}}$ to $\mathcal{W}^{\prime}$ is an isometric isomorphism onto $\mathcal{V}$.

The relationship between the operator $T$ and the derivatives of the $J_{q}(\Gamma)$ is the following.

Theorem 3.7 ([14, Theorem 2.2]). If $\left(h_{1}, \ldots, h_{n}\right) \in \bigoplus_{k=1}^{n} \mathcal{D}_{\text {harm }}\left(\Omega_{k}^{+}\right)$and $q \in$ $\mathfrak{R} \backslash \Gamma$, then

$$
\begin{aligned}
\partial\left[J_{q}(\Gamma)\left(h_{1}, \ldots, h_{n}\right)\right]_{\Sigma} & =-T(\mathcal{O} ; \Sigma)\left(\bar{\partial} h_{1}, \ldots, \bar{\partial} h_{n}\right), \\
\partial\left[J_{q}(\Gamma)\left(h_{1}, \ldots, h_{n}\right)\right]_{\Omega_{j}^{+}} & =-T\left(\mathcal{O} ; \Omega_{j}^{+}\right)\left(\bar{\partial} h_{1}, \ldots, \bar{\partial} h_{n}\right)+\partial h_{j} .
\end{aligned}
$$

For the domain of the Faber operator in the many boundary curve case (Definition 4.1), we define

$$
\overline{\mathcal{D}}(\mathbb{D})^{n}:=\left\{\left(\bar{H}_{1}, \ldots, \bar{H}_{n}\right) \in\left(\overline{\mathcal{D}(\mathbb{D})_{0}}\right)^{n}: \widetilde{\mathcal{C}}_{\mathbf{f}^{-1}}\left(\bar{H}_{1}, \ldots, \bar{H}_{n}\right) \in \mathcal{W}^{\prime}\right\} .
$$

In particular, $\overline{H_{k}}(0)=0$ for each $k$.

The bounce operator $\mathfrak{G}$ (in Section 2.2) can be extended to the many boundary curve case as follows. Let $A_{k}$ be a collar neighbourhood of $\Gamma_{k}$ in $\Omega_{k}^{+}, k=1, \ldots, n$. Let $A=\bigcup A_{k}$. Define $\widetilde{\mathfrak{G}}(A, \mathcal{O})$ by

$$
\begin{aligned}
\widetilde{\mathfrak{G}}(A, \mathcal{O}): \bigoplus_{k=1}^{n} \mathcal{D}\left(A_{k}\right) & \rightarrow \bigoplus_{k=1}^{n} \mathcal{D}_{\text {harm }}\left(\Omega_{k}^{+}\right) \\
\left(h_{1}, \ldots, h_{n}\right) & \rightarrow\left(\mathfrak{G}\left(A_{1}, \Omega_{1}^{+}\right) h_{1}, \ldots, \mathfrak{G}\left(A_{n}, \Omega_{n}^{+}\right) h_{n}\right) .
\end{aligned}
$$

$\widetilde{\mathfrak{G}}(A, \mathcal{O})$ is clearly a bounded operator as $\mathfrak{G}\left(A_{k}, \Omega_{k}^{+}\right)$is bounded for each $k$.

The following lemma will be used to show that $K$ has a left inverse.

Lemma 3.8. Let $\Gamma$ be a quasicircle in $\Re$ separating $\mathfrak{R}$ into two connected subsurfaces $\Sigma_{1}$ and $\Sigma_{2}$. If $A$ is a collar neighbourhood of the curve $\Gamma$ in $\Sigma_{1}$, then for all $h \in \mathcal{D}\left(\operatorname{cl}\left(\Sigma_{2}\right) \cup A\right)$ one has

$$
\mathfrak{O}\left(\Sigma_{2}, \Sigma_{1}\right)\left(\left.h\right|_{\Sigma_{2}}\right)=\mathfrak{G}\left(A, \Sigma_{1}\right)\left(\left.h\right|_{A}\right) .
$$

Proof. By Theorem 2.5 we need to show that both sides of the above equality have the same CNT boundary limits except possibly on a null set in $\Gamma$. The harmonic function $\mathfrak{O}\left(\Sigma_{2}, \Sigma_{1}\right)\left(\left.h\right|_{\Sigma_{2}}\right)$ has the same CNT boundary limits on $\Gamma$ as $\left.h\right|_{\Sigma_{2}}$ has except possibly on a null set in $\Gamma$.

On the other hand, $\mathfrak{G}\left(A, \Sigma_{1}\right)\left(\left.h\right|_{A}\right)$ is the unique element in $\mathcal{D}_{\text {harm }}\left(\Sigma_{1}\right)$ for which its CNT limits equal to $\left.h\right|_{A}$ except possibly on a null set in $\Gamma$. Furthermore, the equality $\left.h\right|_{A}=\left.h\right|_{\Sigma_{2}}$ on $\Gamma$ is clearly true since $h$ is defined on $\operatorname{cl}\left(\Sigma_{2}\right) \cup A$. Therefore, $\mathfrak{O}\left(\Sigma_{2}, \Sigma_{1}\right)\left(\left.h\right|_{\Sigma_{2}}\right)=\mathfrak{G}\left(A, \Sigma_{1}\right)\left(\left.h\right|_{A}\right)$ except possibly on a null set in $\Gamma$ by Lemma 2.4. and they are equal on $\Sigma_{1}$ by Lemma 2.4 and Theorem 2.5

We recall the following theorem (the jump decomposition formula) which is a result of [10, Theorem 4]. Note that because of long formulas in this paper equality always refers to the right hand side of the previous line.

Theorem 3.9 ([14, Theorem 2.12]). For all $\left(\bar{h}_{1}, \ldots, \bar{h}_{n}\right) \in \mathcal{W}^{\prime}$, we have

$$
-\mathfrak{O}\left(\Sigma, \Omega_{j}^{+}\right)\left[J_{q}(\Gamma)\left(\bar{h}_{1}, \ldots, \bar{h}_{n}\right)\right]_{\Sigma}=\bar{h}_{j}-\left[J_{q}(\Gamma)\left(\bar{h}_{1}, \ldots, \bar{h}_{n}\right)\right]_{\Omega_{j}^{+}} .
$$


Theorem 3.10. The operator $-\widetilde{\bar{P}}_{\mathcal{O}} \widetilde{\mathfrak{O}}(\Sigma, \mathcal{O}): \mathcal{D}(\Sigma)_{q} \rightarrow \mathcal{W}^{\prime}$ is a bounded left inverse of $K$.

Proof. Let $\left(\bar{h}_{1}, \ldots, \bar{h}_{n}\right) \in \mathcal{W}^{\prime}$. Then

$$
\begin{aligned}
& -\widetilde{\bar{P}}_{\mathcal{O}} \widetilde{\mathfrak{O}}(\Sigma, \mathcal{O}) K\left(\bar{h}_{1}, \ldots, \bar{h}_{n}\right)=-\widetilde{\bar{P}}_{\mathcal{O}} \widetilde{\mathfrak{O}}(\Sigma, \mathcal{O})\left[J_{q}(\Gamma)\left(\bar{h}_{1}, \ldots, \bar{h}_{n}\right)\right]_{\Sigma} \\
& =-\widetilde{\bar{P}}_{\mathcal{O}}\left(\mathfrak{O}\left(\Sigma, \Omega_{1}^{+}\right)\left[J_{q}(\Gamma)\left(\bar{h}_{1}, \ldots, \bar{h}_{n}\right)\right]_{\Sigma}, \ldots, \mathfrak{O}\left(\Sigma, \Omega_{n}^{+}\right)\left[J_{q}(\Gamma)\left(\bar{h}_{1}, \ldots, \bar{h}_{n}\right)\right]_{\Sigma}\right) \\
& =\widetilde{\widetilde{P}}_{\mathcal{O}}\left(\bar{h}_{1}-\left[J_{q}(\Gamma)\left(\bar{h}_{1}, \ldots, \bar{h}_{n}\right)\right]_{\Omega_{1}^{+}}, \ldots, \bar{h}_{n}-\left[J_{q}(\Gamma)\left(\bar{h}_{1}, \ldots, \bar{h}_{n}\right)\right]_{\Omega_{n}^{+}}\right) \\
& =\left(\bar{h}_{1}, \ldots, \bar{h}_{n}\right)
\end{aligned}
$$

where we have used Theorem 3.9 to show the third equality, and Theorem 3.5 and Definition 3.1 to show the last equality.

The above theorem has an important consequence. Apply projection to both sides of equation (3.1); that is,

$$
-\bar{P}_{\Omega_{j}^{+}} \mathfrak{O}\left(\Sigma, \Omega_{j}^{+}\right)\left[J_{q}(\Gamma)\left(\bar{h}_{1}, \ldots, \bar{h}_{n}\right)\right]_{\Sigma}=\bar{P}_{\Omega_{j}^{+}} \bar{h}_{j}-\bar{P}_{\Omega_{j}^{+}}\left[J_{q}(\Gamma)\left(\bar{h}_{1}, \ldots, \bar{h}_{n}\right)\right]_{\Omega_{j}^{+}} .
$$

Since $\left[J_{q}(\Gamma)\left(\bar{h}_{1}, \ldots, \bar{h}_{n}\right)\right]_{\Omega_{j}^{+}}$is holomorphic (by Theorem 3.5) and $\bar{h}_{j}$ is anti-holomorphic it follows that

$$
-\bar{P}_{\Omega_{j}^{+}} \mathfrak{O}\left(\Sigma, \Omega_{j}^{+}\right)\left[J_{q}(\Gamma)\left(\bar{h}_{1}, \ldots, \bar{h}_{n}\right)\right]_{\Sigma}=\bar{h}_{j} .
$$

So by the definition of the Cauchy-type integral operator one has

$$
-\bar{P}_{\Omega_{j}^{+}} \mathfrak{O}\left(\Sigma, \Omega_{j}^{+}\right) \sum_{k=1}^{n}\left[J_{q}\left(\Gamma_{k}\right)_{\Omega_{k}^{-}} \bar{h}_{k}\right]_{\Sigma}=\bar{h}_{j} .
$$

Note that for fixed $j=1, \ldots, n$ and every $k \neq j$ one has $\Omega_{j}^{+} \subset \Omega_{k}^{-}$, hence the transmission operator can be simplified; that is,

$$
-\bar{P}_{\Omega_{j}^{+}} \sum_{k \neq j}\left[J_{q}\left(\Gamma_{k}\right)_{\Omega_{k}^{-}} \bar{h}_{k}\right]_{\Omega_{j}^{+}}-\bar{P}_{\Omega_{j}^{+}} \mathfrak{O}\left(\Omega_{j}^{-}, \Omega_{j}^{+}\right)\left[J_{q}\left(\Gamma_{j}\right)_{\Omega_{j}^{-}} \bar{h}_{j}\right]=\bar{h}_{j} .
$$

Therefore, for all $\left(\bar{h}_{1}, \ldots, \bar{h}_{n}\right) \in \mathcal{W}^{\prime}$ one has the following important equation:

$$
-\sum_{k \neq j} \bar{P}_{\Omega_{j}^{+}}\left[J_{q}\left(\Gamma_{k}\right)_{\Omega_{k}^{-}} \bar{h}_{k}\right]_{\Omega_{j}^{+}}-\bar{P}_{\Omega_{j}^{+}} \mathfrak{O}\left(\Omega_{j}^{-}, \Omega_{j}^{+}\right)\left[J_{q}\left(\Gamma_{j}\right)_{\Omega_{j}^{-}} \bar{h}_{j}\right]=\bar{h}_{j} .
$$

We also require a density theorem stated and proven in [14, Theorem 3.6].

Theorem 3.11. Let $\mathfrak{R}, \Sigma, \Omega_{k}^{ \pm}, \Gamma_{k}, k=1, \ldots, n$, and let $\mathcal{O}$ be the same as in Section 1. Suppose that each boundary curve $\Gamma_{k}$ is a quasicircle. For each $k=1, \ldots, n$, and let $\epsilon>0$ sufficiently small, define

$$
\Omega_{k \epsilon}^{+}=\left\{w \in \Omega_{k}^{+}: g_{\Omega_{k}^{+}}\left(w, p_{k}\right)<\epsilon\right\}
$$

for some $p_{k}$ fixed in $\Omega_{k}^{+}$. Then the set of the restrictions of functions in $\mathcal{D}(\operatorname{cl}(\Sigma) \cup$ $\left.\Omega_{1 \epsilon}^{+} \cup \cdots \cup \Omega_{n \epsilon}^{+}\right)$to $\Sigma$ is dense in $\mathcal{D}(\Sigma)$. 


\section{FABER OPERATOR}

We are now ready to define the Faber operator. Recall $\mathbf{f}=\left(f_{1}, \ldots, f_{n}\right)$.

Definition 4.1 (Faber operator). Define the Faber operator corresponding to $\mathfrak{R}$ and $\mathbf{f}$ by

$$
\begin{aligned}
\mathbf{I}_{\mathbf{f}}:{\overline{\mathcal{D}_{v}(\mathbb{D})}}^{n} & \rightarrow \mathcal{D}(\Sigma)_{q} \\
\left(\bar{H}_{1}, \ldots, \bar{H}_{n}\right) & \rightarrow-K \widetilde{\mathcal{C}}_{\mathbf{f}^{-1}}\left(\bar{H}_{1}, \ldots, \bar{H}_{n}\right) .
\end{aligned}
$$

In other words, for $z \in \Sigma$, one has

$$
\mathbf{I}_{\mathbf{f}}\left(\bar{H}_{1}, \ldots, \bar{H}_{n}\right)(z)=-\sum_{k=1}^{n}\left[J_{q}\left(\Gamma_{k}\right)_{\Omega_{k}^{-}} \mathcal{C}_{f_{k}^{-1}} \bar{H}_{k}\right]_{\Sigma}(z) .
$$

Corollary 4.2. The Faber operator $\mathbf{I}_{\mathbf{f}}$ is a bounded operator from ${\overline{\mathcal{D}_{v}(\mathbb{D})}}^{n}$ equipped with the norm $\|\cdot\|_{\mathcal{D}_{\text {harm }}(\mathbb{D})^{n}}$ to $\mathcal{D}(\Sigma)_{q}$ equipped with the norm $\|\cdot\|_{\mathcal{D}_{\text {harm }}(\Sigma)}$.

Here is a corollary to Theorem 3.10 .

Corollary 4.3. The operator $\widetilde{\mathcal{C}}_{\mathbf{f}} \widetilde{\bar{P}}_{\mathcal{O}} \widetilde{\mathfrak{O}}(\Sigma, \mathcal{O})$ is a bounded left inverse for the Faber operator $\mathbf{I}_{\mathbf{f}}$. Therefore, $\mathbf{I}_{\mathbf{f}}$ is one-to-one.

Theorem 4.4. Let $\mathfrak{R}, \Sigma, \Gamma_{k}, \Omega_{k}^{ \pm}, f_{k}, k=1, \ldots, n$, and let $\mathcal{O}$ be the same as in Section 1. Assume that all the boundary curves are quasicircles. Then the Faber operator $\mathbf{I}_{\mathbf{f}}: \overline{\mathcal{D}}(\mathbb{D})^{n} \rightarrow \mathcal{D}(\Sigma)_{q}$ is onto.

Proof. Suppose $h \in \mathcal{D}(\Sigma)_{q}$; we then have $\partial h \in A(\Sigma)_{e}$. By the surjectivity of $\left.T(\mathcal{O} ; \Sigma)\right|_{\mathcal{V}}($ Theorem 2.16$)$ there exists $\left(\bar{\alpha}_{1}, \ldots, \bar{\alpha}_{n}\right)$ in $\mathcal{V}$ such that

$$
T(\mathcal{O} ; \Sigma)\left(\bar{\alpha}_{1}, \ldots, \bar{\alpha}_{n}\right)=d h=\partial h \in A(\Sigma)_{e} .
$$

Furthermore by Lemma 3.6 there exists a unique $\left(\bar{h}_{1}, \ldots, \bar{h}_{n}\right) \in \mathcal{W}^{\prime}$ such that $\widetilde{\bar{\partial}}\left(\bar{h}_{1}, \ldots, \bar{h}_{n}\right)=\left(\bar{\alpha}_{1}, \ldots, \bar{\alpha}_{n}\right)$ and

$$
\partial^{-1} T(\mathcal{O} ; \Sigma)\left(\bar{\alpha}_{1}, \ldots, \bar{\alpha}_{n}\right)=-\left[J_{q}(\Gamma)_{\Sigma}\left(\bar{h}_{1}, \ldots, \bar{h}_{n}\right)\right],
$$

or equivalently $h=K\left(-\bar{h}_{1}, \ldots,-\bar{h}_{n}\right)$ by Theorem 3.7. Therefore, for an arbitrary $h \in \mathcal{D}(\Sigma)_{q}$ there exists $\left(-\bar{h}_{1}, \ldots,-\bar{h}_{n}\right) \in \mathcal{W}^{\prime}$ such that

$$
h=K\left(-\bar{h}_{1}, \ldots,-\bar{h}_{n}\right) \text {. }
$$

This proves that $K$ is onto.

On the other hand, $\widetilde{\mathcal{C}}_{\mathbf{f}}-\mathbf{1}$ is a bounded isomorphism (with respect to the Dirichlet semi-norm) which completes the proof.

The following is the first main result of the paper.

Corollary 4.5. Let $\mathfrak{R}, \Sigma, \Gamma_{k}, \Omega_{k}^{ \pm}, f_{k}, k=1, \ldots, n$, and let $\mathcal{O}$ be the same as in Section 1 . If all the boundary curves of $\Sigma$ are quasicircles, then the Faber operator $\mathbf{I}_{\mathbf{f}}: \overline{\mathcal{D}}_{v}(\mathbb{D}){ }^{n} \rightarrow \mathcal{D}(\Sigma)_{q}$ is a bounded isomorphism.

Remark 4.6. Y. L. Shen 19 assigned a Faber operator to a holomorphic map $f$ (of the form $f(z)=z+b_{1} / z+b_{2} / z^{2}+\ldots$, and one-to-one on $\mathbb{D}^{-}$) on $l^{2}(\mathbb{C}$ ). Shen showed that the Faber operator is a bounded isomorphism if and only if $f$ admits a quasiconformal extension to $\mathbb{D}$. E. Schippers and W. Staubach [15] defined a Faber operator corresponding to a biholomorphism map $f$ (from $\mathbb{D}$ to a finite Jordan domain $\Omega^{+}$in $\overline{\mathbb{C}}$ ) on some Dirichlet spaces. They also showed that the Faber operator is a bounded isomorphism precisely for quasiconformally extendible $f$. 


\section{Grunsky operator}

Definition 5.1 (Grunsky operator). Assume all the boundary curves of $\Sigma$ are quasicircles. First, define

$$
G r_{j k}(\mathbf{f}): \overline{\mathcal{D}(\mathbb{D})} \rightarrow \mathcal{D}(\mathbb{D})_{0}
$$

by

$$
G r_{j k}(\mathbf{f}) \bar{H}= \begin{cases}-P_{\mathbb{D}} \mathcal{C}_{f_{j}}\left[J_{q}\left(\Gamma_{k}\right)_{\Omega_{k}^{-}} \mathcal{C}_{f_{k}^{-1}} \bar{H}\right]_{\Omega_{j}^{+}} & \text {if } k \neq j, \\ -P_{\mathbb{D}} \mathcal{C}_{f_{j}} \mathfrak{O}\left(\Omega_{j}^{-}, \Omega_{j}^{+}\right)\left[J_{q}\left(\Gamma_{j}\right)_{\Omega_{j}^{-}} \mathcal{C}_{f_{j}^{-1}} \bar{H}\right] & \text { if } k=j .\end{cases}
$$

Then the Grunsky operator corresponding to $\mathfrak{R}$ and $\mathbf{f}$ is defined by

$$
\begin{aligned}
\mathbf{G r}_{\mathbf{f}}: \overline{\mathcal{D}}(\bar{D})^{n} & \rightarrow \mathcal{D}(\mathbb{D})_{0}^{n} \\
\left(\bar{H}_{1}, \ldots, \bar{H}_{n}\right) & \rightarrow\left(\sum_{k=1}^{n} G r_{1 k}(\mathbf{f}) \bar{H}_{k}, \ldots, \sum_{k=1}^{n} G r_{n k}(\mathbf{f}) \bar{H}_{k}\right) .
\end{aligned}
$$

Remark 5.2. In the case $\mathfrak{g}=0$ this agrees up to some normalizations with 8 , Definition 4.6].

Here is the second main theorem of the paper.

Theorem 5.3. Let $\mathfrak{R}, \Sigma, \Gamma_{k}, \Omega_{k}^{ \pm}, f_{k}, k=1, \ldots, n$, and let $\mathcal{O}$ be as in Section 1 . Assume all the boundary curves of $\Sigma$ are quasicircles. Then the Grunsky operator

$$
\begin{aligned}
\mathbf{G r}_{\mathbf{f}}:\left(\overline{\mathcal{D}}_{v}(\mathbb{D})^{n},\|\cdot\|_{\mathcal{D}_{\text {harm }}(\mathbb{D})^{n}}\right) & \longrightarrow\left(\mathcal{D}(\mathbb{D})_{0}^{n},\|\cdot\|_{\mathcal{D}_{\text {harm }}(\mathbb{D})^{n}}\right) \\
\left(\bar{H}_{1}, \ldots, \bar{H}_{n}\right) & \longrightarrow\left(\sum_{k=1}^{n} G r_{1 k}(\mathbf{f}) \bar{H}_{k}, \ldots, \sum_{k=1}^{n} G r_{n k}(\mathbf{f}) \bar{H}_{k}\right)
\end{aligned}
$$

is a bounded operator of norm less than or equal to one. Furthermore one has

$$
\begin{aligned}
& \left\|\mathbf{I}_{\mathbf{f}}\left(\bar{H}_{1}, \ldots, \bar{H}_{n}\right)\right\|_{\mathcal{D}_{\text {harm }}(\Sigma)}^{2} \\
& \quad=-\left\|\mathbf{G r}_{\mathbf{f}}\left(\bar{H}_{1}, \ldots, \bar{H}_{n}\right)\right\|_{\mathcal{D}_{\text {harm }}(\mathbb{D})^{n}}^{2}+\left\|\left(\bar{H}_{1}, \ldots, \bar{H}_{n}\right)\right\|_{\mathcal{D}_{\text {harm }}(\mathbb{D})^{n}}^{2} .
\end{aligned}
$$

To prove the above we require a few lemmas. We start with the following lemma which has a straightforward proof.

Lemma 5.4. Let $\Omega$ be a simply connected domain in a Riemann surface. Let $f: \mathbb{D} \rightarrow \Omega$ be a biholomorphism such that $f(0)=p$ for some $p \in \Omega$. Then

$$
\bar{P}_{\mathbb{D}} \mathcal{C}_{f}=\mathcal{C}_{f} \bar{P}_{\Omega}
$$

Lemma 5.5. Let $\Gamma$ be a strip-cutting Jordan curve on a compact Riemann surface $\mathfrak{R}$ dividing $\mathfrak{R}$ into two connected subsurfaces $\Sigma$ and $\Omega$. Assume $\Omega$ is simply connected. Let $f: \mathbb{D} \rightarrow \Omega$ be a biholomorphism onto $\Omega$ and let $\mathbb{A}$ be an annulus in $\mathbb{D}$ whose outer boundary is $\mathbb{S}^{1}$. Suppose $f$ maps $\mathbb{A}$ biholomorphically onto a collar neighbourhood $A=f(\mathbb{A})$ of $\Gamma$ in $\Omega$. Then for $h \in \mathcal{D}_{\text {harm }}(A)$ one has

$$
\mathfrak{G}(\mathbb{A}, \mathbb{D}) \mathcal{C}_{f} h=\mathcal{C}_{f} \mathfrak{G}(A, \Omega) h .
$$

Proof. First note that the operator $\mathcal{C}_{f}$ on the left hand side is in fact $\mathcal{C}_{\left.f\right|_{\mathbb{A}}}$; we, however, use the same notation as $\mathcal{C}_{f}$ for simplicity. The existence of the CNT limits of $h$ on $\Gamma$ follows from Theorem 2.6. 
If $h \in \mathcal{D}_{\text {harm }}(A)$, then by definition of $\mathfrak{G}(\mathbb{A}, \mathbb{D}), \mathfrak{G}(\mathbb{A}, \mathbb{D}) \mathcal{C}_{f} h$ is the unique function in $\mathcal{D}_{\text {harm }}(\mathbb{D})$ such that $\mathfrak{G}(\mathbb{A}, \mathbb{D}) \mathcal{C}_{f} h$ and $\mathcal{C}_{f} h$ have the same non-tangential limits on $\mathbb{S}^{1}$ except possibly on a set of logarithmic capacity zero in $\mathbb{S}^{1}$.

Similarly, $\mathfrak{G}(A, \Omega) h$ is the unique function in $\mathcal{D}_{\text {harm }}(\Omega)$ such that $\mathfrak{G}(A, \Omega) h$ and $h$ have the same CNT boundary values on $\Gamma$ except possibly on a null set in $\Gamma$. By Definition 2.2 this means that $\mathcal{C}_{f} \mathfrak{G}(A, \Omega) h$ and $\mathcal{C}_{f} h$ have the same non-tangential limits on $\mathbb{S}^{1}$ except possibly on a set of logarithmic capacity zero in $\mathbb{S}^{1}$.

Therefore, $\mathfrak{G}(\mathbb{A}, \mathbb{D}) \mathcal{C}_{f} h$ and $\mathcal{C}_{f} \mathfrak{G}(A, \Omega) h$ have the same non-tangential limits on $\mathbb{S}^{1}$ except possibly on a finite union of sets of logarithmic capacity zero in $\mathbb{S}^{1}$, so they are equal on $\mathbb{D}$ by Lemma 2.4 .

Lemma 5.6 (Laurent decomposition). Let $\mathbb{A}$ be an annulus with outer boundary equal to $\mathbb{S}^{1}$. A function $h \in \mathcal{D}(\mathbb{A})$ can be decomposed to $h_{+}+h_{-}$where

$$
\begin{aligned}
& h_{+}=P_{\mathbb{D}} \mathfrak{G}(\mathbb{A}, \mathbb{D}) h \in \mathcal{D}(\mathbb{D}), \\
& h_{-}=\mathfrak{O}\left(\mathbb{D}, \mathbb{D}^{-}\right) \bar{P}_{\mathbb{D}} \mathfrak{G}(\mathbb{A}, \mathbb{D}) h \in \mathcal{D}\left(\mathbb{D}^{-}\right) .
\end{aligned}
$$

Proof. Note that $\mathfrak{G}(\mathbb{A}, \mathbb{D}) z^{n}=z^{n}$ for $n \geq 1, \mathfrak{G}(\mathbb{A}, \mathbb{D}) z^{-n}=\bar{z}^{n}$ for $n \geq 0, \mathfrak{O}\left(\mathbb{D}, \mathbb{D}^{-}\right) z^{n}$ $=\left(\frac{1}{\bar{z}}\right)^{n}$ for $n \geq 1$, and $\mathfrak{O}\left(\mathbb{D}, \mathbb{D}^{-}\right) \bar{z}^{n}=\left(\frac{1}{z}\right)^{n}$ for $n \geq 0$. Then the density of the polynomials in $z^{n}$ and $z^{-n}$ in the Dirichlet space $\mathcal{D}(\mathbb{A})$ and the boundedness of the $\mathfrak{O}$ and $\mathfrak{G}$ operators completes the proof.

Lemma 5.7. The transmission operator $\mathfrak{O}\left(\mathbb{D}, \mathbb{D}^{-}\right)$has norm one so it is an isometry with respect to the Dirichlet semi-norm. That is,

$$
\left\|\mathfrak{O}\left(\mathbb{D}, \mathbb{D}^{-}\right) h\right\|_{\mathcal{D}_{\text {harm }}\left(\mathbb{D}^{-}\right)}=\|h\|_{\mathcal{D}_{\text {harm }}(\mathbb{D})}
$$

for every $h \in \mathcal{D}_{\text {harm }}(\mathbb{D})$. A similar result is true for $\mathfrak{O}\left(\mathbb{D}^{-}, \mathbb{D}\right)$.

Proof. It can be shown that this is true for monomials. Thus the identity follows by the density of these elements in $\mathcal{D}_{\text {harm }}(\mathbb{D})$ and the boundedness of the transmission operator.

Lemma 5.8. Let $H$ and $G$ be holomorphic functions on $\mathbb{D}$. Then

$$
\begin{aligned}
& \int_{\gamma_{r}}\left(\mathfrak{O}\left(\mathbb{D}, \mathbb{D}^{-}\right) \bar{H}\right)^{\prime}(z) \bar{G}(z) d z=0, \\
& \int_{\gamma_{r}}\left(\overline{\mathfrak{O}\left(\mathbb{D}, \mathbb{D}^{-}\right) \bar{H}}\right)(z) G^{\prime}(z) d z=0,
\end{aligned}
$$

where $\gamma_{r}=\{z \in \mathbb{C}:|z|=r\}$ for $0<r<1$.

The above lemma can be proven by using the Taylor expansion of the functions inside the integrals. We now give the proof of Theorem 5.3

Proof. (Theorem 5.3). We first prove the claim for the set of all $\left(\bar{H}_{1}, \ldots, \bar{H}_{n}\right)$ in $\overline{\mathcal{D}}(\mathbb{D})^{n}$ such that $\mathbf{I}_{\mathbf{f}}\left(\bar{H}_{1}, \ldots, \bar{H}_{n}\right) \in \mathcal{D}(\Sigma)_{q}$ has a holomorphic extension past the boundary of $\Sigma$. More precisely, we assume that $\mathbf{I}_{\mathbf{f}}\left(\bar{H}_{1}, \ldots, \bar{H}_{n}\right)$ carries a holomorphic extension to a collar neighbourhood $A_{k}$ of the boundary curve $\Gamma_{k}$ in $\Omega_{k}^{+}$for each $k=1, \ldots, n$. The proof on this set of functions is rather straightforward. Then by the density theorem, Theorem 3.11 and the boundedness of the operators involved in the proof, the claim holds for every element in $\mathcal{D}(\Sigma)_{q}$.

To apply the density theorem assume $A_{k}=\Omega_{k \epsilon}^{+}$for some sufficiently small $\epsilon>0$ depending on $\left(\bar{H}_{1}, \ldots, \bar{H}_{n}\right)$. By the above assumption and the finiteness of the 
number of the curves, there is a number $R \in(0,1)$ such that the image of $\gamma_{R}=$ $\{z \in \mathbb{C}:|z|=R\}$ under $f_{k}$ is entirely in $A_{k}$ for all $k=1, \ldots, n$. Let $\mathbb{A}=$ $\{z \in \mathbb{C}: R<|z|<1\}$. Shrink the $A_{k}$ 's (but keep using the same notation) so that $\left.f_{k}\right|_{\mathbb{A}}: \mathbb{A} \rightarrow A_{k}$ is a biholomorphism, $k=1, \ldots, n$. Then $f\left(\gamma_{r}\right), r \in(R, 1)$, are simple closed analytic curves in $\Omega_{k \epsilon}^{+}$which approach $\Gamma_{k}$ from within $\Omega_{k \epsilon}^{+}$as $r$ approaches one. By Green's identity and the fact that $\Gamma_{k}$ 's are negatively oriented with respect to surface $\Sigma$ it follows that

$$
\begin{aligned}
&\left\|\mathbf{I}_{\mathbf{f}}\left(\bar{H}_{1}, \ldots, \bar{H}_{n}\right)\right\|_{\mathcal{D}_{\text {harm }}(\Sigma)}^{2} \\
&= \iint_{\Sigma}\left|\mathbf{I}_{\mathbf{f}}\left(\bar{H}_{1}, \ldots, \bar{H}_{n}\right)^{\prime}\right|^{2} \\
&=-\frac{1}{2 i} \lim _{r \rightarrow 1^{-}} \int_{\cup f_{j}\left(\gamma_{r}\right)} \mathbf{I}_{\mathbf{f}}\left(\bar{H}_{1}, \ldots, \bar{H}_{n}\right)^{\prime}(z) \overline{\mathbf{I}_{\mathbf{f}}\left(\bar{H}_{1}, \ldots, \bar{H}_{n}\right)(z)} d z \\
&=-\frac{1}{2 i} \lim _{r \rightarrow 1^{-}} \sum_{j=1}^{n} \int_{f_{j}\left(\gamma_{r}\right)} \mathbf{I}_{\mathbf{f}}\left(\bar{H}_{1}, \ldots, \bar{H}_{n}\right)^{\prime}(z) \overline{\mathbf{I}_{\mathbf{f}}\left(\bar{H}_{1}, \ldots, \bar{H}_{n}\right)(z)} d z \\
&=-\frac{1}{2 i} \lim _{r \rightarrow 1^{-}} \sum_{j=1}^{n} \int_{f_{j}\left(\gamma_{r}\right)}\left(-\sum_{k=1}^{n}\left[J_{q}\left(\Gamma_{k}\right)_{\Omega_{k}^{-}} \mathcal{C}_{f_{k}^{-1}} \bar{H}_{k}\right]_{A_{j}}(z)\right)^{\prime} \\
& \times\left(-\sum_{k=1}^{n}\left[J_{q}\left(\Gamma_{k}\right)_{\Omega_{k}^{-}} \mathcal{C}_{f_{k}^{-1}} \bar{H}_{k}\right]_{A_{j}}(z)\right) d z \\
&=-\frac{1}{2 i} \lim _{r \rightarrow 1^{-}} \sum_{j=1}^{n} \int_{\gamma_{r}}\left(-\sum_{k=1}^{n}\left[J_{q}\left(\Gamma_{k}\right)_{\Omega_{k}^{-}} \mathcal{C}_{f_{k}^{-1}} \bar{H}_{k}\right]_{A_{j}}\left(f_{j}(w)\right)\right)^{\prime} \\
& \times\left(-\sum_{k=1}^{n}\left[J_{q}\left(\Gamma_{k}\right)_{\Omega_{k}^{-}} \mathcal{C}_{f_{k}^{-1}} \bar{H}_{k}\right]_{A_{j}}\left(f_{j}(w)\right)\right) d w .
\end{aligned}
$$

We used $\left(\Omega_{j}^{+}, f_{j}\right)$ as holomorphic charts to set up the integrals on the Riemann surface $\mathfrak{R}$, which defines the variable $z_{j}=f_{j}\left(w_{j}\right)$ in each term. For simplicity we denote $z=z_{j}$, and $w=w_{j}$. By $h^{\prime}$ for a function $h$ on $\Omega_{j}^{+}$, we mean $\partial h / \partial z$ in the holomorphic chart $\left(\Omega_{j}^{+}, f_{j}\right)$. We continue simplifying the above integral using the composition operator. $\mathcal{C}_{f_{j}}$ here is $\mathcal{C}_{\left.f_{j}\right|_{\AA}}$. The above is equal to

$$
\begin{aligned}
& -\frac{1}{2 i} \lim _{r \rightarrow 1^{-}} \sum_{j=1}^{n} \int_{\gamma_{r}}\left(-\mathcal{C}_{f_{j}} \sum_{k=1}^{n}\left[J_{q}\left(\Gamma_{k}\right)_{\Omega_{k}^{-}} \mathcal{C}_{f_{k}^{-1}} \bar{H}_{k}\right]_{A_{j}}(w)\right)^{\prime} \\
& \quad \times\left(-\mathcal{C}_{f_{j}} \sum_{k=1}^{n}\left[J_{q}\left(\Gamma_{k}\right)_{\Omega_{k}^{-}} \mathcal{C}_{f_{k}^{-1}} \bar{H}_{k}\right]_{A_{j}}(w)\right) d w
\end{aligned}
$$

The assumption of the existence of a holomorphic extension of $\mathbf{I}_{\mathbf{f}}$ past the boundary of $\Sigma$ implies that for fixed $j=1, \ldots, n$, the sum $\sum_{k=1}^{n}\left[J_{q}\left(\Gamma_{k}\right)_{\Omega_{k}^{-}} \mathcal{C}_{f_{k}^{-1}} \bar{H}_{k}\right]$ has a holomorphic extension past the boundary curve $\Gamma_{j}$ to $A_{j}$. For $k \neq j$, each term $J_{q}\left(\Gamma_{k}\right)_{\Omega_{k}^{-}} \mathcal{C}_{f_{k}^{-1}} \bar{H}_{k}$ in the sum automatically admits such a holomorphic extension to $A_{j}$ by its definition. So our assumption, in fact, implies that $J_{q}\left(\Gamma_{j}\right)_{\Omega_{j}^{-}} \mathcal{C}_{f_{j}^{-1}} \bar{H}_{j}$ admits a holomorphic extension to $A_{j}$. 
We proceed by simplifying the integrand in (5.2). The choice of annulus $\mathbb{A}$ implies that $-\mathcal{C}_{f_{j}} \sum_{k=1}^{n}\left[J_{q}\left(\Gamma_{k}\right)_{\Omega_{k}^{-}} \mathcal{C}_{f_{k}^{-1}} \bar{H}_{k}\right]_{A_{j}} \in \mathcal{D}(\mathbb{A})$. Therefore, by applying Lemma [5.6, we have the identity

$$
\begin{aligned}
& -\mathcal{C}_{f_{j}} \sum_{k=1}^{n}\left[J_{q}\left(\Gamma_{k}\right)_{\Omega_{k}^{-}} \mathcal{C}_{f_{k}^{-1}} \bar{H}_{k}\right]_{A_{j}} \\
& =\left[\mathfrak{O}\left(\mathbb{D}, \mathbb{D}^{-}\right) \bar{P}_{\mathbb{D}} \mathfrak{G}(\mathbb{A}, \mathbb{D})\left(-\mathcal{C}_{f_{j}} \sum_{k=1}^{n}\left[J_{q}\left(\Gamma_{k}\right)_{\Omega_{k}{ }^{-}} \mathcal{C}_{f_{k}^{-1}} \bar{H}_{k}\right]_{A_{j}}\right)\right] \\
& \quad+\left[P_{\mathbb{D}} \mathfrak{G}(\mathbb{A}, \mathbb{D})\left(-\mathcal{C}_{f_{j}} \sum_{k=1}^{n}\left[J_{q}\left(\Gamma_{k}\right)_{\Omega_{k}^{-}} \mathcal{C}_{f_{k}^{-1}} \bar{H}_{k}\right]_{A_{j}}\right)\right]
\end{aligned}
$$

By applying Lemma [5.5 and splitting the series into two parts, the above is

$$
\begin{aligned}
- & {\left[\mathfrak{O}\left(\mathbb{D}, \mathbb{D}^{-}\right) \bar{P}_{\mathbb{D}} \mathcal{C}_{f_{j}} \mathfrak{G}\left(A_{j}, \Omega_{j}^{+}\right)\left(\sum_{k \neq j}\left[J_{q}\left(\Gamma_{k}\right)_{\Omega_{k}} \mathcal{C}_{f_{k}^{-1}} \bar{H}_{k}\right]_{A_{j}}\right)\right] } \\
- & {\left[\mathfrak{O}\left(\mathbb{D}, \mathbb{D}^{-}\right) \bar{P}_{\mathbb{D}} \mathcal{C}_{f_{j}} \mathfrak{G}\left(A_{j}, \Omega_{j}^{+}\right)\left[J_{q}\left(\Gamma_{j}\right)_{\Omega_{j}^{-}} \mathcal{C}_{f_{j}^{-1}} \bar{H}_{j}\right]_{A_{j}}\right] } \\
- & {\left[P_{\mathbb{D}} \mathcal{C}_{f_{j}} \mathfrak{G}\left(A_{j}, \Omega_{j}^{+}\right)\left(\sum_{k \neq j}\left[J_{q}\left(\Gamma_{k}\right)_{\Omega_{k}^{-}} \mathcal{C}_{f_{k}^{-1}} \bar{H}_{k}\right]_{A_{j}}\right)\right] } \\
- & {\left[P_{\mathbb{D}} \mathcal{C}_{f_{j}} \mathfrak{G}\left(A_{j}, \Omega_{j}^{+}\right)\left[J_{q}\left(\Gamma_{j}\right)_{\Omega_{j}^{-}} \mathcal{C}_{f_{j}^{-}} \bar{H}_{j}\right]_{A_{j}}\right] . }
\end{aligned}
$$

Lemma 3.8 (let $\Sigma_{1}=\Omega_{j}^{+}, \Sigma_{2}=\Omega_{j}^{-}$) and the definition of $\mathfrak{G}\left(A_{j}, \Omega_{j}^{+}\right)$(or Theorem 2.5) imply that the above is equal to

$$
\begin{aligned}
- & {\left[\mathfrak{O}\left(\mathbb{D}, \mathbb{D}^{-}\right) \bar{P}_{\mathbb{D}} \mathcal{C}_{f_{j}} \sum_{k \neq j}\left[J_{q}\left(\Gamma_{k}\right)_{\Omega_{k}^{-}} \mathcal{C}_{f_{k}^{-1}} \bar{H}_{k}\right]_{\Omega_{j}^{+}}\right] } \\
- & {\left[\mathfrak{O}\left(\mathbb{D}, \mathbb{D}^{-}\right) \bar{P}_{\mathbb{D}} \mathcal{C}_{f_{j}} \mathfrak{O}\left(\Omega_{j}^{-}, \Omega_{j}^{+}\right)\left[J_{q}\left(\Gamma_{j}\right)_{\Omega_{j}^{-}} \mathcal{C}_{f_{j}^{-1}} \bar{H}_{j}\right]_{\Omega_{j}^{-}}\right] } \\
- & {\left[P_{\mathbb{D}} \mathcal{C}_{f_{j}} \sum_{k \neq j}\left[J_{q}\left(\Gamma_{k}\right)_{\Omega_{k}^{-}} \mathcal{C}_{f_{k}^{-1}} \bar{H}_{k}\right]_{\Omega_{j}^{+}}\right] } \\
& -\left[P_{\mathbb{D}} \mathcal{C}_{f_{j}} \mathfrak{O}\left(\Omega_{j}^{-}, \Omega_{j}^{+}\right)\left[J_{q}\left(\Gamma_{j}\right)_{\Omega_{j}^{-}} \mathcal{C}_{f_{j}^{-1}} \bar{H}_{j}\right]_{\Omega_{j}^{-}}\right]
\end{aligned}
$$

By Lemma 5.4 (note that $f_{j}(0)=p_{j}, j=1, \ldots, n$ ) and Definition 5.1 , the above is

$$
\begin{aligned}
\mathfrak{O}\left(\mathbb{D}, \mathbb{D}^{-}\right) \mathcal{C}_{f_{j}}\left[-\sum_{k \neq j}\left(\bar{P}_{\Omega_{j}^{+}}\left[J_{q}\left(\Gamma_{k}\right)_{\Omega_{k}^{-}} \mathcal{C}_{f_{k}^{-1}} \bar{H}_{k}\right]_{\Omega_{j}^{+}}\right)\right. \\
\left.\quad-\bar{P}_{\Omega_{j}^{+}} \mathfrak{O}\left(\Omega_{j}^{-}, \Omega_{j}^{+}\right)\left[J_{q}\left(\Gamma_{j}\right)_{\Omega_{j}^{-}} \mathcal{C}_{f_{j}^{-1}} \bar{H}_{j}\right]_{\Omega_{j}^{-}}\right] \\
\quad+\sum_{k \neq j} G r_{j k}(\mathbf{f}) \bar{H}_{k}+G r_{j j}(\mathbf{f}) \bar{H}_{j} .
\end{aligned}
$$


We know that $\left(\mathcal{C}_{f_{1}^{-1}} \bar{H}_{1}, \ldots, \mathcal{C}_{f_{n}^{-1}} \bar{H}_{n}\right) \in \mathcal{W}^{\prime}$, so applying equation (3.3) implies that the above is

$$
\begin{aligned}
& \mathfrak{O}\left(\mathbb{D}, \mathbb{D}^{-}\right) \mathcal{C}_{f_{j}}\left[-\sum_{k \neq j}\left(\bar{P}_{\Omega_{j}^{+}}\left[J_{q}\left(\Gamma_{k}\right)_{\Omega_{k}^{-}} \mathcal{C}_{f_{k}^{-1}} \bar{H}_{k}\right]_{\Omega_{j}^{+}}\right)\right. \\
& \left.\quad-\bar{P}_{\Omega_{j}^{+}} \mathfrak{O}\left(\Omega_{j}^{-}, \Omega_{j}^{+}\right)\left[J_{q}\left(\Gamma_{j}\right)_{\Omega_{j}^{-}} \mathcal{C}_{f_{j}^{-1}} \bar{H}_{j}\right]_{\Omega_{j}^{-}}\right]+\sum_{k=1}^{n} G r_{j k}(\mathbf{f}) \bar{H}_{k} \\
& =\mathfrak{O}\left(\mathbb{D}, \mathbb{D}^{-}\right) \mathcal{C}_{f_{j}}\left(\mathcal{C}_{f_{j}^{-1}} \bar{H}_{j}\right)+\sum_{k=1}^{n} G r_{j k}(\mathbf{f}) \bar{H}_{k} . \\
& =\mathfrak{O}\left(\mathbb{D}, \mathbb{D}^{-}\right) \bar{H}_{j}+\sum_{k=1}^{n} G r_{j k}(\mathbf{f}) \bar{H}_{k} .
\end{aligned}
$$

Therefore, we obtained the following important identity:

$$
-\mathcal{C}_{f_{j}} \sum_{k=1}^{n}\left[J_{q}\left(\Gamma_{k}\right)_{\Omega_{k}^{-}} \mathcal{C}_{f_{k}^{-1}} \bar{H}_{k}\right]_{A_{j}}=\mathfrak{O}\left(\mathbb{D}, \mathbb{D}^{-}\right) \bar{H}_{j}+\sum_{k=1}^{n} G r_{j k}(\mathbf{f}) \bar{H}_{k} .
$$

Now by inserting equation (5.3) in (5.2) and also by the extension property of $\mathfrak{O}\left(\mathbb{D}, \mathbb{D}^{-}\right) \bar{H}_{j}$ explained in Remark 2.8 , we have

$$
\begin{aligned}
& \left\|\mathbf{I}_{\mathbf{f}}\left(\bar{H}_{1}, \ldots, \bar{H}_{n}\right)\right\|_{\mathcal{D}_{\text {harm }}(\Sigma)}^{2} \\
& =-\frac{1}{2 i} \lim _{r \rightarrow 1^{-}} \sum_{j=1}^{n} \int_{\gamma_{r}}\left(\mathfrak{O}\left(\mathbb{D}, \mathbb{D}^{-}\right) \bar{H}_{j}(w)+\sum_{k=1}^{n} G r_{j k}(\mathbf{f}) \bar{H}_{k}(w)\right)^{\prime} \\
& \times\left(\overline{\mathfrak{O}\left(\mathbb{D}, \mathbb{D}^{-}\right) \bar{H}_{j}(w)+\sum_{k=1}^{n} G r_{j k}(\mathbf{f}) \bar{H}_{k}(w)}\right) d w \\
& =-\frac{1}{2 i} \lim _{r \rightarrow 1^{-}} \sum_{j=1}^{n} \int_{\gamma_{r}}\left(\mathfrak{O}\left(\mathbb{D}, \mathbb{D}^{-}\right) \bar{H}_{j}(w)\right)^{\prime}\left(\overline{\mathfrak{O}\left(\mathbb{D}, \mathbb{D}^{-}\right) \bar{H}_{j}(w)}\right) d w \\
& -\frac{1}{2 i} \lim _{r \rightarrow 1^{-}} \sum_{j=1}^{n} \int_{\gamma_{r}}\left(\sum_{k=1}^{n} G r_{j k}(\mathbf{f}) \bar{H}_{k}(w)\right)^{\prime}\left(\overline{\sum_{k=1}^{n} G r_{j k}(\mathbf{f}) \bar{H}_{k}(w)}\right) d w \\
& -\frac{1}{2 i} \lim _{r \rightarrow 1^{-}} \sum_{j=1}^{n} \int_{\gamma_{r}}\left(\mathfrak{O}\left(\mathbb{D}, \mathbb{D}^{-}\right) \bar{H}_{j}(w)\right)^{\prime}\left(\overline{\sum_{k=1}^{n} G r_{j k}(\mathbf{f}) \bar{H}_{k}(w)}\right) d w \\
& -\frac{1}{2 i} \lim _{r \rightarrow 1^{-}} \sum_{j=1}^{n} \int_{\gamma_{r}}\left(\sum_{k=1}^{n} G r_{j k}(\mathbf{f}) \bar{H}_{k}(w)\right)^{\prime}\left(\overline{\mathfrak{O}\left(\mathbb{D}, \mathbb{D}^{-}\right) \bar{H}_{j}(w)}\right) d w .
\end{aligned}
$$

The first integral in the above identity can be simplified as follows:

$$
\begin{aligned}
& -\frac{1}{2 i} \lim _{r \rightarrow 1^{-}} \sum_{j=1}^{n} \int_{\gamma_{r}}\left(\mathfrak{O}\left(\mathbb{D}, \mathbb{D}^{-}\right) \bar{H}_{j}(w)\right)^{\prime}\left(\overline{\mathfrak{O}\left(\mathbb{D}, \mathbb{D}^{-}\right) \bar{H}_{j}(w)}\right) d w \\
& =\sum_{j=1}^{n}\left\|\mathfrak{O}\left(\mathbb{D}, \mathbb{D}^{-}\right) \bar{H}_{j}\right\|_{\mathcal{D}_{\text {harm }}\left(\mathbb{D}^{-}\right)}^{2} \\
& =\sum_{j=1}^{n}\left\|\bar{H}_{j}\right\|_{\mathcal{D}_{\text {harm }}(\mathbb{D})}^{2},
\end{aligned}
$$


where we have used Lemma 5.7, the Green's identity, and the fact that $\mathfrak{O}\left(\mathbb{D}, \mathbb{D}^{-}\right) \bar{H}_{j}$ is in $\mathcal{D}_{\text {harm }}\left(\operatorname{cl}\left(\mathbb{D}^{-}\right) \cup \mathbb{A}\right)$, and $\gamma_{r}$ 's are negatively oriented with respect to $\operatorname{cl}\left(\mathbb{D}^{-}\right) \cup \mathbb{A}$.

For the second term in (5.4), we similarly have

$$
\begin{aligned}
& -\frac{1}{2 i} \lim _{r \rightarrow 1^{-}} \sum_{j=1}^{n} \int_{\gamma_{r}}\left(\sum_{k=1}^{n} G r_{j k}(\mathbf{f}) \bar{H}_{k}(w)\right)^{\prime}\left(\overline{\sum_{k=1}^{n} G r_{j k}(\mathbf{f}) \bar{H}_{k}(w)}\right) d w \\
& =-\sum_{j=1}^{n} \iint_{\mathbb{D}}\left|\left(\sum_{k=1}^{n} G r_{j k}(\mathbf{f}) \bar{H}_{k}(w)\right)^{\prime}\right|^{2} d w=-\sum_{j=1}^{n}\left\|\sum_{k=1}^{n} G r_{j k}(\mathbf{f}) \bar{H}_{k}\right\|_{\mathcal{D}_{\text {harm }}(\mathbb{D})}^{2},
\end{aligned}
$$

as $\gamma_{r}$ 's are positively oriented with respect to the domain of integration.

Now consider the fourth term in (5.4)

$$
-\frac{1}{2 i} \lim _{r \rightarrow 1^{-}} \sum_{j=1}^{n} \int_{\gamma_{r}}\left(\sum_{k=1}^{n} G r_{j k}(\mathbf{f}) \bar{H}_{k}(w)\right)^{\prime}\left(\overline{\mathfrak{O}\left(\mathbb{D}, \mathbb{D}^{-}\right) \bar{H}_{j}(w)}\right) d w=0,
$$

by Lemma 5.8 and the fact that $G_{j}:=\sum_{k=1}^{n} G r_{j k}(\mathbf{f}) \overline{H_{k}}$ is holomorphic on $\mathbb{D}$. Applying Lemma 5.8 one more time shows that the third term in (5.4) is also zero. Therefore, (5.4) becomes

$$
\begin{aligned}
& =-\sum_{j=1}^{n}\left\|\sum_{k=1}^{n} G r_{j k}(\mathbf{f}) \bar{H}_{k}\right\|_{\mathcal{D}_{\text {harm }}(\mathbb{D})}^{2}+\sum_{j=1}^{n}\left\|\bar{H}_{j}\right\|_{\mathcal{D}_{\text {harm }}(\mathbb{D})}^{2} \\
& =-\left\|\mathbf{G r}_{\mathbf{f}}\left(\bar{H}_{1}, \ldots, \bar{H}_{n}\right)\right\|_{\mathcal{D}_{\text {harm }}(\mathbb{D})^{n}}^{2}+\left\|\left(\bar{H}_{1}, \ldots, \bar{H}_{n}\right)\right\|_{\mathcal{D}_{\text {harm }}(\mathbb{D})^{n}}^{2} .
\end{aligned}
$$

Finally, by the above equation and (5.2) we have the following identity:

$$
\begin{aligned}
& \left\|\mathbf{I}_{\mathbf{f}}\left(\bar{H}_{1}, \ldots, \bar{H}_{n}\right)\right\|_{\mathcal{D}_{\text {harm }}(\Sigma)}^{2} \\
& \quad=-\left\|\mathbf{G r}_{\mathbf{f}}\left(\bar{H}_{1}, \ldots, \bar{H}_{n}\right)\right\|_{\mathcal{D}_{\text {harm }}(\mathbb{D})^{n}}^{2}+\left\|\left(\bar{H}_{1}, \ldots, \bar{H}_{n}\right)\right\|_{\mathcal{D}_{\text {harm }}(\mathbb{D})^{n}}^{2} .
\end{aligned}
$$

This completes the proof for those $\left(\bar{H}_{1}, \ldots, \bar{H}_{n}\right) \in \overline{\mathcal{D}}(\mathbb{D})^{n}$ for which $\mathbf{I}_{\mathbf{f}}\left(\bar{H}_{1}, \ldots, \bar{H}_{n}\right)$ admits a holomorphic extension past the boundary of $\Sigma$.

By Theorem 3.11 and the boundedness of the operators used here one has the identity (5.1) for all elements in ${\overline{\mathcal{D}_{v}(\mathbb{D})}}^{n}$; this completes the proof.

The proof of the norm of the Grunsky operator is strictly less than one when all the boundary curves are quasicircles is a consequence of the fact that the Faber operator is a bounded isomorphism.

Theorem 5.9. Let $\mathfrak{R}, \Sigma, \Gamma_{k}, \Omega_{k}^{ \pm}, f_{k}, k=1, \ldots, n$, and let $\mathcal{O}$ be as in Section 1 , If all the boundary curves are quasicircles, then the norm of the Grunsky operator is strictly less than one.

Proof. By Corollary 4.5 there exists a constant $0<c<1$ such that

$$
c\left\|\left(\bar{H}_{1}, \ldots, \bar{H}_{n}\right)\right\|_{\mathcal{D}_{\text {harm }}(\mathbb{D})^{n}} \leq\left\|\mathbf{I}_{\mathbf{f}}\left(\bar{H}_{1}, \ldots, \bar{H}_{n}\right)\right\|_{\mathcal{D}_{\text {harm }}(\Sigma)} .
$$

Now the identity (5.1) and the above inequality complete the proof.

Remark 5.10. It is well known that the norm of the Grunsky operator for a planar domain $G$ is less than one precisely when the boundary of $G$ is a quasicircle (i.e., $G$ is a quasidisc); see Pommerenke [6, Sec 3.1 and 9.4]. 
6. Characterization of $\mathcal{C}_{f} \mathcal{D}(\Sigma)$ as the graph of the Grunsky operator Let $\left(a_{k}\right)_{k=1}^{n}$ denote the $n$-tuple $\left(a_{1}, \ldots, a_{n}\right)$. Here is our third main result.

Theorem 6.1. Let $\mathfrak{R}, \Sigma, \Gamma_{k}, \Omega_{k}^{ \pm}, f_{k}, k=1, \ldots, n$, and let $\mathcal{O}$ be as in Section 1 . Assume all the boundary curves are quasicircles. Then

$$
\widetilde{\mathcal{C}_{\mathbf{f}}} \widetilde{\mathfrak{O}}(\Sigma, \mathcal{O}) \mathcal{D}(\Sigma)_{q}=\operatorname{graph}\left(\mathbf{G r}_{\mathbf{f}}\right)
$$

In other words, the set of pull-back of the transmission of functions in $\mathcal{D}(\Sigma)_{q}$ under $\mathbf{f}=\left(f_{1}, \ldots, f_{n}\right)$ is the graph of the Grunsky operator $\mathbf{G r}_{\mathbf{f}}$.

Proof. By Corollary 4.5, we know that $\mathbf{I}_{\mathbf{f}}$ is a bounded isomorphism from $\overline{\mathcal{D}}(\mathbb{D})^{n}$ onto $\mathcal{D}(\Sigma)_{q}$. We split the proof into two steps.

Step 1. We calculate the composition of $\widetilde{\mathfrak{O}}(\Sigma, \mathcal{O})$ with $\mathbf{I}_{\mathbf{f}}\left(\bar{H}_{1}, \ldots, \bar{H}_{n}\right)$. Then

$$
\begin{aligned}
& \widetilde{\mathfrak{O}}(\Sigma, \mathcal{O}) \mathbf{I}_{\mathbf{f}}\left(\bar{H}_{1}, \ldots, \bar{H}_{n}\right)=\widetilde{\mathfrak{O}}(\Sigma, \mathcal{O})\left(-\sum_{k=1}^{n}\left[J_{q}\left(\Gamma_{k}\right)_{\Omega_{k}^{-}} \mathcal{C}_{f_{k}^{-1}} \bar{H}_{k}\right]_{\Sigma}\right) \\
& =-\left(\mathfrak{O}\left(\Sigma, \Omega_{j}^{+}\right) \sum_{k=1}^{n}\left[J_{q}\left(\Gamma_{k}\right)_{\Omega_{k}^{-}} \mathcal{C}_{f_{k}^{-1}} \bar{H}_{k}\right]_{\Sigma}\right)_{j=1}^{n} \\
& =-\left(\sum_{\substack{k=1 \\
k \neq j}}^{n}\left[J_{q}\left(\Gamma_{k}\right)_{\Omega_{k}^{-}} \mathcal{C}_{f_{k}^{-1}} \bar{H}_{k}\right]_{\Omega_{j}^{+}}+\mathfrak{O}\left(\Omega_{j}^{-}, \Omega_{j}^{+}\right)\left[J_{q}\left(\Gamma_{j}\right)_{\Omega_{j}^{-}} \mathcal{C}_{f_{j}^{-1}} \bar{H}_{j}\right]\right)_{j=1}^{n},
\end{aligned}
$$

where $\mathfrak{O}\left(\Sigma, \Omega_{j}^{+}\right) \sum_{k=1}^{n}\left[J_{q}\left(\Gamma_{k}\right)_{\Omega_{k}^{-}} \mathcal{C}_{f_{k}^{-1}} \bar{H}_{k}\right]_{\Sigma}$ in the second line is the $j$ th component of $n$-tuple $\widetilde{\mathfrak{O}}(\Sigma, \mathcal{O}) \mathbf{I}_{\mathbf{f}}\left(\bar{H}_{1}, \ldots, \bar{H}_{n}\right)$.

The identity above can be justified as follows. Note that for fixed $j=1, \ldots, n$, if $k \neq j$, the function $J_{q}\left(\Gamma_{k}\right)_{\Omega_{k}^{-}} \mathcal{C}_{f_{k}^{-1}} \bar{H}_{k}$ is harmonic on $\Omega_{j}^{+} \subset \Omega_{k}^{-}$. Therefore, by Definition 3.2 the transmission through $\Gamma_{j}$ of this term to $\Omega_{j}^{+}$is itself. For $k=j$, the function $J_{q}\left(\Gamma_{j}\right)_{\Omega_{j}^{-}} \mathcal{C}_{f_{j}^{-1}} \bar{H}_{j}$ is defined and is harmonic on $\Omega_{j}^{-}$; this implies that

$$
\begin{aligned}
\mathfrak{O}\left(\Sigma, \Omega_{j}^{+}\right)\left[J_{q}\left(\Gamma_{j}\right)_{\Omega_{j}^{-}} \mathcal{C}_{f_{j}^{-1}} \bar{H}_{j}\right]_{\Sigma} & =\mathfrak{O}\left(\Omega_{j}^{-}, \Omega_{j}^{+}\right) \mathfrak{G}\left(A_{j}, \Omega_{j}^{-}\right)\left[J_{q}\left(\Gamma_{j}\right)_{\Omega_{j}^{-}} \mathcal{C}_{f_{j}^{-1}} \bar{H}_{j}\right]_{A_{j}} \\
& =\mathfrak{O}\left(\Omega_{j}^{-}, \Omega_{j}^{+}\right)\left[J_{q}\left(\Gamma_{j}\right)_{\Omega_{j}^{-}} \mathcal{C}_{f_{j}^{-1}} \bar{H}_{j}\right],
\end{aligned}
$$

where $A_{j}$ is defined in the same way as Definition 3.2 , for each $j=1, \ldots, n$. The second identity above is by Theorem 2.5. 
Step 2. Now we calculate $\widetilde{P}_{\mathbb{D}} \widetilde{\mathcal{C}_{\mathbf{f}}} \widetilde{\mathfrak{O}}(\Sigma, \mathcal{O}) \mathbf{I}_{\mathbf{f}}$ and $\widetilde{P_{\mathbb{D}}} \widetilde{\mathcal{C}_{\mathbf{f}}} \widetilde{\mathfrak{O}}(\Sigma, \mathcal{O}) \mathbf{I}_{\mathbf{f}}$. Let $\left(\bar{H}_{1}, \ldots, \bar{H}_{n}\right)$ be in ${\overline{\mathcal{D}_{v}(\mathbb{D})}}^{n}$. Then

$$
\begin{aligned}
& {\left[\widetilde{P}_{\mathbb{D}} \widetilde{\mathcal{C}_{\mathbf{f}}} \widetilde{\mathfrak{O}}(\Sigma, \mathcal{O}) \mathbf{I}_{\mathbf{f}}\right]\left(\bar{H}_{1}, \ldots, \bar{H}_{n}\right)=\widetilde{P}_{\mathbb{D}} \widetilde{\mathcal{C}_{\mathbf{f}}} \widetilde{\mathfrak{O}}(\Sigma, \mathcal{O})\left(-\sum_{k=1}^{n}\left[J_{q}\left(\Gamma_{k}\right)_{\Omega_{k}^{-}} \mathcal{C}_{f_{k}^{-1}} \bar{H}_{k}\right]_{\Sigma}\right)} \\
& =-\widetilde{P}_{\mathbb{D}} \widetilde{\mathcal{C}_{\mathbf{f}}}\left(\sum_{\substack{k=1 \\
k \neq j}}^{n}\left[J_{q}\left(\Gamma_{k}\right)_{\Omega_{k}^{-}} \mathcal{C}_{f_{k}^{-1}} \bar{H}_{k}\right]_{\Omega_{j}^{+}}+\mathfrak{O}\left(\Omega_{j}^{-}, \Omega_{j}^{+}\right)\left[J_{q}\left(\Gamma_{j}\right)_{\Omega_{j}^{-}} \mathcal{C}_{f_{j}^{-1}} \bar{H}_{j}\right]\right)_{j=1}^{n} \\
& =-\widetilde{P}_{\mathbb{D}}\left(\sum_{\substack{k=1 \\
k \neq j}}^{n} \mathcal{C}_{f_{j}}\left[J_{q}\left(\Gamma_{k}\right)_{\Omega_{k}^{-}} \mathcal{C}_{f_{k}^{-1}} \bar{H}_{k}\right]_{\Omega_{j}^{+}}+\mathcal{C}_{f_{j}} \mathfrak{O}\left(\Omega_{j}^{-}, \Omega_{j}^{+}\right)\left[J_{q}\left(\Gamma_{j}\right)_{\Omega_{j}^{-}} \mathcal{C}_{f_{j}^{-1}} \bar{H}_{j}\right]\right)_{j=1}^{n} \\
& =\left(\sum_{\substack{k=1 \\
k \neq j}}^{n}\left(-P_{\mathbb{D}} \mathcal{C}_{f_{j}}\left[J_{q}\left(\Gamma_{k}\right)_{\Omega_{k}^{-}} \mathcal{C}_{f_{k}^{-1}} \bar{H}_{k}\right]_{\Omega_{j}^{+}}\right)-P_{\mathbb{D}} \mathcal{C}_{f_{j}} \mathfrak{D}\left(\Omega_{j}^{-}, \Omega_{j}^{+}\right)\left[J_{q}\left(\Gamma_{j}\right)_{\Omega_{j}^{-}} \mathcal{C}_{f_{j}^{-1}} \bar{H}_{j}\right]\right)_{j=1}^{n} \\
& =\left(\sum_{\substack{k=1 \\
k \neq j}}^{n} G r_{j k}(\mathbf{f}) \bar{H}_{k}+G r_{j j}(\mathbf{f}) \bar{H}_{j}\right)_{j=1}^{n}=\left(\sum_{k=1}^{n} G r_{j k}(\mathbf{f}) \bar{H}_{k}\right)_{j=1}^{n} \\
& =\left(\sum_{k=1}^{n} G r_{1 k}(\mathbf{f}) \bar{H}_{k}, \ldots, \sum_{k=1}^{n} G r_{n k}(\mathbf{f}) \bar{H}_{k}\right)=\mathbf{G r}_{\mathbf{f}}\left(\bar{H}_{1}, \ldots, \bar{H}_{n}\right) \text {. }
\end{aligned}
$$

Therefore, we obtained the identity $\widetilde{P_{\mathbb{D}}} \widetilde{\mathcal{C}_{\mathbf{f}}} \widetilde{\mathfrak{O}}(\Sigma, \mathcal{O}) \mathbf{I}_{\mathbf{f}}=\mathbf{G r}_{\mathbf{f}}$.

On the other hand, by applying Lemma 5.4 to each map $f_{j}$, we have

$$
\begin{aligned}
{\left[\widetilde{\bar{P}}_{\mathbb{D}} \widetilde{\mathcal{C}_{\mathbf{f}}} \widetilde{\mathfrak{D}}(\Sigma, \mathcal{O}) \mathbf{I}_{\mathbf{f}}\right]\left(\bar{H}_{1}, \ldots, \bar{H}_{n}\right) } & =\widetilde{\bar{P}}_{\mathbb{D}} \widetilde{\mathcal{C}_{\mathbf{f}}} \widetilde{\mathfrak{O}}(\Sigma, \Omega)\left(-K \widetilde{\mathcal{C}}_{\mathbf{f}^{-1}}\left(\bar{H}_{1}, \ldots, \bar{H}_{n}\right)\right) \\
& =\widetilde{\bar{P}}_{\mathbb{D}} \widetilde{\mathcal{C}}_{\mathbf{f}}\left(-\mathfrak{O}\left(\Sigma, \Omega_{j}^{+}\right) K \widetilde{\mathcal{C}}_{\mathbf{f}^{-1}}\left(\bar{H}_{1}, \ldots, \bar{H}_{n}\right)\right)_{j=1}^{n} \\
& =\widetilde{\bar{P}}_{\mathbb{D}}\left(-\mathcal{C}_{f_{j}} \mathfrak{O}\left(\Sigma, \Omega_{j}^{+}\right) K \widetilde{\mathcal{C}}_{\mathbf{f}^{-1}}\left(\bar{H}_{1}, \ldots, \bar{H}_{n}\right)\right)_{j=1}^{n} \\
& =\left(-\bar{P}_{\mathbb{D}} \mathcal{C}_{f_{j}} \mathfrak{O}\left(\Sigma, \Omega_{j}^{+}\right) K \widetilde{\mathcal{C}}_{\mathbf{f}^{-1}}\left(\bar{H}_{1}, \ldots, \bar{H}_{n}\right)\right)_{j=1}^{n} \\
& =\left(-\mathcal{C}_{f_{j}} \bar{P}_{\Omega_{j}^{+}} \mathfrak{O}\left(\Sigma, \Omega_{j}^{+}\right) K \widetilde{\mathcal{C}}_{\mathbf{f}^{-1}}\left(\bar{H}_{1}, \ldots, \bar{H}_{n}\right)\right)_{j=1}^{n} \\
& =\left(\mathcal{C}_{f_{j}}\left(\mathcal{C}_{f_{j}^{-1}} \bar{H}_{j}\right)\right)_{j=1}^{n}=\left(\bar{H}_{1}, \ldots, \bar{H}_{n}\right) \\
& =I_{\overline{\mathcal{D}}_{v}(\mathbb{D})}{ }^{n}\left(\bar{H}_{1}, \ldots, \bar{H}_{n}\right),
\end{aligned}
$$

where the sixth equality is a result of equation (3.2). Therefore,

$$
I_{\overline{\mathcal{D}}_{v}(\mathbb{D})}^{n}=\widetilde{\bar{P}}_{\mathbb{D}} \widetilde{\mathcal{C}_{\mathbf{f}}} \widetilde{\mathfrak{O}}(\Sigma, \Omega) \mathbf{I}_{\mathbf{f}}
$$


Finally, by the above two equalities proven in Step 2 and equation (2.1) it follows that

$$
\begin{aligned}
\widetilde{\mathcal{C}_{\mathbf{f}}} \widetilde{\mathfrak{D}} & (\Sigma, \Omega) \mathbf{I}_{\mathbf{f}}\left(\bar{H}_{1}, \ldots, \bar{H}_{n}\right) \\
& =\left[\left(\widetilde{P}_{\mathbb{D}}+\widetilde{\bar{P}}_{\mathbb{D}}\right) \widetilde{\mathcal{C}_{\mathbf{f}}} \widetilde{\mathfrak{D}}(\Sigma, \Omega) \mathbf{I}_{\mathbf{f}}\right]\left(\bar{H}_{1}, \ldots, \bar{H}_{n}\right) \\
& =\left[\widetilde{P}_{\mathbb{D}} \widetilde{\mathcal{C}_{\mathbf{f}}} \widetilde{\mathfrak{D}}(\Sigma, \Omega) \mathbf{I}_{\mathbf{f}}\right]\left(\bar{H}_{1}, \ldots, \bar{H}_{n}\right)+\left[\widetilde{\bar{P}}_{\mathbb{D}} \widetilde{\widetilde{\mathcal{C}}_{\mathbf{f}}} \widetilde{\mathfrak{D}}(\Sigma, \Omega) \mathbf{I}_{\mathbf{f}}\right]\left(\bar{H}_{1}, \ldots, \bar{H}_{n}\right) \\
& =\mathbf{G r}_{\mathbf{f}}\left(\bar{H}_{1}, \ldots, \bar{H}_{n}\right)+I_{\overline{\mathcal{D}}_{v}(\mathbb{D})}{ }^{n}\left(\bar{H}_{1}, \ldots, \bar{H}_{n}\right)
\end{aligned}
$$

which is in $\operatorname{graph}\left(\mathbf{G r}_{\mathbf{f}}\right)$. This completes the proof.

Remark 6.2. The result of the paper for the Riemann sphere and $n=0$ or $n=1$ reduces to those of [8, Theorem 4.1] and [8, Theorem 4.10].

\section{ACKNOWLEDGMENTS}

The author would like to thank his supervisor, Eric Schippers, for introducing the group of ideas discussed here and his valuable comments on the paper. The author is also grateful to the referee for the time and helpful comments. Finally, the author is grateful to NSERC and the University of Manitoba for financial support.

\section{REFERENCES}

[1] Lars V. Ahlfors and Leo Sario, Riemann surfaces, Princeton Mathematical Series, No. 26, Princeton University Press, Princeton, N.J., 1960. MR0114911

[2] S. Bergman and M. Schiffer, Kernel functions and conformal mapping, Compositio Math. 8 (1951), 205-249. MR39812

[3] Omar El-Fallah, Karim Kellay, Javad Mashreghi, and Thomas Ransford, A primer on the Dirichlet space, Cambridge Tracts in Mathematics, vol. 203, Cambridge University Press, Cambridge, 2014. MR3185375

[4] H. M. Farkas and I. Kra, Riemann surfaces, 2nd ed., Graduate Texts in Mathematics, vol. 71, Springer-Verlag, New York, 1992. MR1139765

[5] Olli Lehto, Univalent functions and Teichmüller spaces, Graduate Texts in Mathematics, vol. 109, Springer-Verlag, New York, 1987. MR867407

[6] C. Pommerenke, Univalent Functions, no. 229 in Grundlehren der mathematischen Wissenschaften, Springer-Verlag, 1975.

[7] D. Radnell, E. Schippers, M. Shirazi, and W. Staubach, Schiffer Operator and Calculation of a Determinant Line in Conformal Field Theory, submitted.

[8] David Radnell, Eric Schippers, and Wolfgang Staubach, Dirichlet spaces of domains bounded by quasicircles, Commun. Contemp. Math. 22 (2020), no. 3, 1950022, 22, DOI 10.1142/S0219199719500226. MR4082224

[9] H. L. Royden, Harmonic functions on open Riemann surfaces, Trans. Amer. Math. Soc. 73 (1952), 40-94, DOI 10.2307/1990823. MR49396

[10] H. L. Royden, Function theory on compact Riemann surfaces, J. Analyse Math. 18 (1967), 295-327, DOI 10.1007/BF02798051. MR214757

[11] Menahem Schiffer, The kernel function of an orthonormal system, Duke Math. J. 13 (1946), 529-540. MR 19115

[12] M. Schiffer and G. Schober, Coefficient problems and generalized Grunsky inequalities for schlicht functions with quasiconformal extensions, Arch. Rational Mech. Anal. 60 (1975/76), no. 3, 205-228, DOI 10.1007/BF01789257. MR404609

[13] Menahem Schiffer and Donald C. Spencer, Functionals of finite Riemann surfaces, Princeton University Press, Princeton, N. J., 1954. MR0065652

[14] E. Schippers, M. Shirazi, and W. Staubach, Schiffer comparison operators and approximations on Riemann surfaces bordered by quasicircles, to appear in J. Geom. Anal.

[15] Eric Schippers and Wolfgang Staubach, Riemann boundary value problem on quasidisks, Faber isomorphism and Grunsky operator, Complex Anal. Oper. Theory 12 (2018), no. 2, 325-354, DOI 10.1007/s11785-016-0598-4. MR3756161 
[16] E. Schippers and W. Staubach, Plemelj-Sokhotski isomorphism for quasicircles in Riemann surfaces and the Schiffer operators, Math. Ann. doi:10.1007/s00208-019-01922-4, 2019.

[17] Eric Schippers and Wolfgang Staubach, Transmission of harmonic functions through quasicircles on compact Riemann surfaces, Ann. Acad. Sci. Fenn. Math. 45 (2020), no. 2, 11111134, DOI 10.5186/aasfm.2020.4559. MR4112278

[18] Hiroshige Shiga, On analytic and geometric properties of Teichmüller spaces, J. Math. Kyoto Univ. 24 (1984), no. 3, 441-452, DOI 10.1215/kjm/1250521274. MR.766636

[19] YuLiang Shen, Faber polynomials with applications to univalent functions with quasiconformal extensions, Sci. China Ser. A 52 (2009), no. 10, 2121-2131, DOI 10.1007/s11425-0090062-2. MR2550270

[20] Yu-liang Shen, On Grunsky operator, Sci. China Ser. A 50 (2007), no. 12, 1805-1817, DOI 10.1007/s11425-007-0141-1. MR2390490

[21] Yuliang Shen, The asymptotic Teichmüller space and the asymptotic Grunsky map, Proc. Roy. Soc. Edinburgh Sect. A 140 (2010), no. 3, 651-672, DOI 10.1017/S0308210509000560. MR.2651378

[22] Leon A. Takhtajan and Lee-Peng Teo, Weil-Petersson metric on the universal Teichmüller space, Mem. Amer. Math. Soc. 183 (2006), no. 861, viii+119, DOI 10.1090/memo/0861. MR.2251887

[23] I. V. Zuravlev, Univalent functions and Teichmüller spaces (Russian), Dokl. Akad. Nauk SSSR 250 (1980), no. 5, 1047-1050. MR561570

Department of Mathematics, University of Manitoba, Winnipeg, Canada

Email address: shirazim@myumanitoba.ca

Email address: mohammad.shirazi@mail.mcgill.ca 